\title{
Cognition-Enhancing Vagus Nerve Stimulation Alters the Epigenetic Landscape
}

\author{
DTeresa H. Sanders, ${ }^{1,2,3}$ Joseph Weiss, ${ }^{1}$ Luke Hogewood, ${ }^{2}$ Lan Chen, ${ }^{3}$ Casey Paton, ${ }^{1}$ Rebekah L. McMahan, ${ }^{1}$ \\ and $\odot$ J. David Sweatt ${ }^{1}$ \\ ${ }^{1}$ Department of Pharmacology, 2Department of Biomedical Engineering, and ${ }^{3}$ Neuroscience Program, Vanderbilt University, Nashville, Tennessee 37232
}

\begin{abstract}
Vagus nerve stimulation (VNS) has been shown to enhance learning and memory, yet the mechanisms behind these enhancements are unknown. Here, we present evidence that epigenetic modulation underlies VNS-induced improvements in cognition. We show that VNS enhances novelty preference (NP); alters the hippocampal, cortical, and blood epigenetic transcriptomes; and epigenetically modulates neuronal plasticity and stress-response signaling genes in male Sprague Dawley rats. Brain-behavior analysis revealed structure-specific relationships between NP test performance (NPTP) and epigenetic alterations. In the hippocampus, NPTP correlated with decreased histone deacetylase 11 (HDAC11), a transcriptional repressor enriched in CA1 cells important for memory consolidation. In the cortex, the immediate early gene (IEG) ARC was increased in VNS rats and correlated with transcription of plasticity genes and epigenetic regulators, including HDAC3. For rats engaged in NPTP, ARC correlated with performance. Interestingly, blood ARC transcripts decreased in VNS rats performing NPTP, but increased in VNS-only rats. Because DNA double-strand breaks (DSBs) facilitate transcription of IEGs, we investigated phosphorylated H2A.X ( $\gamma \mathrm{H} 2 \mathrm{~A} . \mathrm{X})$, a histone modification known to colocalize with DSBs. In agreement with reduced cortical stress-response transcription factor NF- $\kappa B 1$, chromatin immunoprecipitation revealed reduced $\gamma \mathrm{H} 2 \mathrm{~A}$.X in the ARC promoter. Surprisingly, VNS did not significantly reduce transcription of cortical or hippocampal proinflammatory cytokines. However, TNFRSF11B (osteoprotegerin) correlated with NPTP as well as plasticity, stress-response signaling, and epigenetic regulation transcripts in both hippocampus and cortex. Together, our findings provide the first evidence that VNS induces widespread changes in the cognitive epigenetic landscape and specifically affects epigenetic modulators associated with NPTP, stress-response signaling, memory consolidation, and cortical neural remodeling.
\end{abstract}

Key words: chromatin; DNA double-strand breaks; epigenetic; learning and memory; plasticity; vagus nerve stimulation

Significance Statement

Recent studies have implicated vagus nerve stimulation (VNS) in enhanced learning and memory. However, whereas epigenetic modifications are known to play an important role in memory, the particular mechanisms involved in VNS-enhanced cognition are unknown. In this study, we examined brain and behavior changes in VNS and sham rats performing a multiday novelty preference (NP) task. We found that VNS activated specific histone modifications and DNA methylation changes at important stress-response signaling and plasticity genes. Both cortical and hippocampal plasticity changes were predictive of NP test performance. Our results reveal important epigenetic alterations associated with VNS cognitive improvements, as well as new potential pharmacological targets for enhancing cortical and hippocampal plasticity.

\section{Introduction}

Vagus nerve stimulation (VNS) has been shown to facilitate plasticity and memory in animal models and humans (Clark et al.,

Received Sept. 13, 2018; revised Feb. 15, 2019; accepted Feb. 16, 2019.

Author contributions: T.H.S. designed research; T.H.S., J.W., L.H., L.C., C.P., and R.L.M. performed research; T.H.S. and L.H. analyzed data; T.H.S. wrote the first draft of the paper; T.H.S. and J.D.S. edited the paper; T.H.S. wrote the paper.

This work was sponsored by the Defense Advanced Research Projects Agency (DARPA) Biological Technologies Office (BTO) Targeted Neuroplasticity Training (TNT) program under the auspices of Dr. Tristan McClure-Begley (SPAWAR Cooperative Agreement N66001-17-2-4019). We thank Timothy Broderick for intellectual support throughout the design and implementation of these experiments; Lindsey Noble, Kris Loerwald, Kim Rahebi, Seth
1999; Hays et al., 2014; Alvarez-Dieppa et al., 2016; Borland et al., 2016; Cao et al., 2016; Sun et al., 2017). However, although the acetylcholine- and norepinephrine-driven immunomodulatory
Hays, and Mike Kilgard at UT Dallas for training THS in vagus nerve cuff construction and surgical implantation; Ben Litt for assistance with cuff building; and Katie Sanders for brain tissue (qRT-PCR) and bioinformatic data extraction. The views, opinions, and/or findings contained in this article are those of the authors and should not be interpreted as representing the official views or policies of the Department of Defense or the U.S. Government.

All sequencing data that support the findings of this study have been deposited in the National Center for Biotechnology Information Gene Expression Omnibus (GE0) and are accessible through GEO. All other relevant data are available from the corresponding author on request.

The authors declare no competing financial interests. 
effects of VNS have been thoroughly examined (Borovikova et al., 2000; Berridge and Waterhouse, 2003; Roosevelt et al., 2006; Rosas-Ballina et al., 2011; Andersson and Tracey, 2012; Hulsey et al., 2017; Patel et al., 2017), the epigenetic mechanisms underlying VNS-enhanced learning and memory have not yet been studied. Here, we investigated how VNS facilitates memory formation and consolidation through epigenetic mechanisms of histone modifications (Levenson et al., 2004; Itzhak et al., 2013; Zentner and Henikoff, 2013), DNA methylation (Levenson et al., 2006; Miller et al., 2008; Feng et al., 2010, Meadows et al., 2015; Jones, 2012), and RNA changes in hippocampus, cortex, and blood.

Learning activates waves of altered gene expression in the hippocampus (Levenson et al., 2004; Lubin et al., 2008; Bekinschtein et al., 2007). Early changes in gene expression initiate memory formation and then further changes occur $12-24 \mathrm{~h}$ after training to enable memory persistence through the process of systems consolidation (Frankland et al., 2006; Bekinschtein et al., 2007). During systems consolidation, memories are uploaded from the hippocampus to the cortex for persistent storage (Levenson et al., 2004; Miller et al., 2010; Itzhak et al., 2013; Zentner and Henikoff, 2013). Epigenetic mechanisms such as chromatin remodeling, DNA methylation, and RNA-DNA-protein interactions are critical for effective learning and memory (Levenson et al., 2006; Miller et al., 2008, 2010; Day and Sweatt, 2010; Feng et al., 2010; Jones, 2012; Kaas et al., 2013; Meadows et al., 2015; Kennedy and Sweatt, 2016; Kennedy et al., 2016; Savell et al., 2016). Chromatin remodeling affects early memory formation by modulating gene transcription and also controls persistent memory storage by increasing or decreasing access to cortical chromatin (Levenson et al., 2004; Lubin et al., 2008; Day and Sweatt, 2011; Itzhak et al., 2013; Sweatt, 2013; Zentner and Henikoff, 2013; Zovkic et al., 2013). Chromatin remodeling typically involves modifications to histone tails such as phosphorylation or acetylation (both increase access to DNA), methylation, or ubiquitination. DNA methyltransferases (DNMTs) regulate cytosine methylation at cytosine-guanine dinucleotide rich-sequences within DNA (CpG islands), thereby altering local chromatin structure and the transcription of local plasticity genes (Levenson et al., 2006; Miller et al., 2008, 2010; Feng et al., 2010; Jones, 2012; Meadows et al., 2015).

The intertwining of memory and epigenetic mechanisms has inspired efforts to identify an "epigenetic code" or "histone code" that enumerates the relationships between epigenetic modulators and synaptic plasticity, learning, and memory (Turner, 2000; Rosenfeld et al., 2006; Zovkic et al., 2013). Indeed, many relationships between epigenetic effects and the underlying modulation of transcription and translation have been identified. For example, the histone modification $\mathrm{H} 3 \mathrm{~K} 4 \mathrm{me} 3$ (trimethylation of histone $\mathrm{H} 3$ on lysine 4) is known to be associated with increased transcriptional activation (Dai et al., 2018) and the exchange of canonical histone subunit $2 \mathrm{~A}(\mathrm{H} 2 \mathrm{~A})$ with histone variant $\mathrm{H} 2 \mathrm{~A} . \mathrm{X}$ is known to play a role in signaling DNA damage events (Pinto and Flaus, 2010; Sharma et al., 2012) such as double-strand breaks (DSBs), as well as providing a foundation for the assembly of repair machinery such as ligases and cofactors.

In this study, we show that VNS enhances novelty preference (NP) during a multiday object interaction task and investigate whether epigenetic mechanisms underlie the enhancement.

Correspondence should be addressed to Teresa H. Sanders at teresa.hinkle.sanders@gmail.com. https://doi.org/10.1523/JNEUROSCI.2407-18.2019

Copyright $\odot 2019$ the authors
Through joint behavioral and transcriptome analysis with specific epigenetic assays for DNA methylation (bisulfite conversion/pyrosequencing) and histone modifications (chromatin immunoprecipitation, ChIP), we identify VNS-induced epigenetic and transcriptome modifications associated with enhanced cognition, reduced stress-response signaling, cortical synaptic plasticity, calcium signaling changes, and increased hippocampal output.

\section{Materials and Methods}

Subjects. All experiments were approved by the Vanderbilt University Animal Research Committee. Thirty-five adult male Sprague Dawley rats (4-5 months old; $350 \pm 50 \mathrm{~g})$ were used for this study.

VNS device construction. VNS cuff electrodes were built based on the designs used in previous studies (Engineer et al., 2011). Briefly, the cuff of the bipolar stimulating electrode is constructed from $4 \mathrm{~mm}$ of MicroRenethane tubing ( $1.8 \mathrm{~mm}$ inner diameter). The electrodes are made of coated multistranded platinum iridium wire $/{ }^{\prime \prime}(0.006$ "). The region in contact with the nerve is stripped of insulation for $8 \mathrm{~mm}$ and attached to the inner surface of the cuff separated by $2 \mathrm{~mm}$. Silk thread is used to enable closing the cuff edges during surgery. A head cap consisting of a four-channel Omnetics plug soldered to gold pins is connected to the VNS cuff wire leads during surgery.

VNS surgical protocol. Before surgery, rats were given buprenorphine $(0.03 \mathrm{mg} / \mathrm{kg}$, i.p. $)$ as preemptive analgesia. Animals were then anesthetized with ketamine/xylazine and placed in a stereotaxic apparatus (Kopf Instruments). The head cap with the four-channel Omnetics plug was attached to the skull with screws and dental cement. VNS rats were then implanted with a bipolar cuff electrode around the left cervical vagus nerve. As in humans, only the left vagus nerve was stimulated because the right vagus nerve innervates the sinoatrial node and can cause cardiac complications. Leads from the electrode were tunneled subcutaneously to the top of the head and affixed to the head cap. Sham rats received an identical surgery including installation of a head cap with plug, exposure of the left cervical vagus nerve (Alvarez-Dieppa et al., 2016), and creation of a subcutaneous tunnel from the nerve site to the head cap as in VNS rats. However, for sham rats, no cuff was implanted.

Calibrating and confirming effects of VNS on heart rate, oxygen saturation, and behavior. To ensure that cuffs were operating as expected based on previous studies (Alvarez-Dieppa et al., 2016; Borland et al., 2016), we verified that $30 \mathrm{~s}$ of VNS at $60 \mathrm{~Hz}$ and $0.8 \mathrm{~mA}$ transiently decreased the blood oxygen saturation and/or reduced the breathing rate in all VNS implanted rats. However, the standard intermittent $30 \mathrm{~Hz}$ VNS protocol used in this study had no measurable effect on either heart rate or oxygen saturation. These results are consistent with our visual observations that intermittent VNS bursts as specified in our protocol cause no noticeable behavioral response or change in heart rate or breathing.

VNS stimulation protocol. A four-channel connector from the stimulus generator was plugged into each rat's head cap before all VNS and sham stimulation sessions. Cuff impedances were measured before and after implantation to ensure that all cuffs fell within the appropriate range. Stimulation parameters used in the current study were based on previous plasticity studies (Clark et al., 1999; Engineer et al., 2011). Stimulation parameters were identical for all VNS rats in the study. Specifically, charge-balanced biphasic pulses with amplitude $0.8 \mathrm{~mA}$ were delivered as a train of $15100 \mu$ s pulses at $30 \mathrm{~Hz}$ (500 ms train duration). Stimulation trains were delivered every $17.5 \mathrm{~s}$ over a $30 \mathrm{~min}$ period. Sham rats were connected to the stimulus generator in the same way as VNS rats except they received no stimulation.

Behavioral testing. To measure the effects of VNS on cognition stimulated and sham rats $(N=12, n=7 \mathrm{VNS}$ and $n=5$ sham, cohort 3$)$ were tested using NP tasks $1 \mathrm{~d}$ after receiving VNS (or sham VNS) during object familiarization. Previous studies in rats have found the NP task, also referred to as the novel object recognition task, to be very useful for studying short-term memory, immediate-term memory, and long-term memory (Broadbent et al., 2010; Gaskin et al., 2010; Haettig et al., 2011; Antunes and Biala, 2012). In our study, we used four objects: two different rope/bead rat toys purchased at a pet store, a jar lid, and a Lego toy 
car. After an initial habituation session in the open field, rats were allowed to become familiar with the first object on day 1 during administration of $30 \mathrm{~min}$ of intermittent VNS bursts (see Fig. 1A). On day 2, the first object was placed in its original location and a second object was placed in the opposite corner of the open field. Rats were tested for 10 min to determine whether they preferred the novel (second) object over the familiar (first) object. As in previous studies, cognitive enhancement was assessed by calculating the difference in the amount of time spent interacting with the novel object $\left(t_{\mathrm{o} 2}\right)$ compared with the familiar object $\left(t_{\mathrm{o} 1}\right)$, expressed as a fraction of the total time spent interacting with either object during the test as follows: (Haettig et al., 2011)

$$
\left(\text { novel object preference }=\frac{t_{o 2}-t_{o 1}}{t_{o 2}+t_{o 1}}\right)
$$

Immediately following the $10 \mathrm{~min}$ NP test, rats received $30 \mathrm{~min}$ of VNS in the arena, enabling familiarization with the objects from that day's NP task. On days 3 and 4, the "oldest" object was removed, the object introduced on the previous day became the familiar object (o1), and an unfamiliar novel object (o2) was added. As on day 2, rats performed the 10 min NP test and then immediately received 30 min of VNS while in the arena. Note that both the sham and VNS rats had stimulation cables continuously attached to their head caps for the entirety of the 10 min test and 30 min stimulation phases. The use of a commutator ensured that the rats could move freely about the arena.

Behavioral data extraction. Rats were videotaped while performing the NP task. Start and ending times of stimulation were recorded in a log book. Videotapes were then reviewed by blinded analysts who recorded time stamps and durations when rats interacted with the learned object (o1) and the novel object (o2). Interactions were categorized as proximity, whisking, nudging, pawing, biting, and combinations of interactions. Each rat's novel object preference measures were calculated based on the summed total times of interaction for each of the two objects during the 10 min testing period for each day.

Nucleic acid isolation. For all experiments, animals were killed by rapid decapitation after completion of day 1 (cohort 1 ) or day 4 (cohorts 2 and 3 ) of stimulation. Brains were immediately removed and dissected. Brain regions were sectioned, flash frozen on dry ice, and stored at $-80^{\circ} \mathrm{C}$. Cortical and hippocampal sections were obtained from $3 \mathrm{~mm}(\mathrm{~A} / \mathrm{P})$ coronal brain sections centered on $-3.5 \mathrm{~mm}$ (bregma). Trunk blood was collected in PAXgene blood RNA tubes, kept at room temperature for $24 \mathrm{~h}$, and then frozen. Tissue samples were processed for nucleic acid isolation using the AllPrep DNA/RNA/miRNA Universal Kit (Qiagen). Blood was processed with the Invitrogen Ribopure blood kit. Nucleic acid concentrations were determined using a Nanodrop spectrophotometer (Thermo Scientific).

$q R T-P C R$. Total RNA was split into aliquots for qRT-PCR and RNA sequencing (RNA-seq). qRT-PCR aliquots were reverse transcribed using the iScript RT-PCR kit (Bio-Rad). qRT-PCR amplifications were performed in triplicate using an iQ5 real-time PCR system (Bio-Rad) at $95^{\circ} \mathrm{C}$ for $5 \mathrm{~min}$, followed by 40 cycles of $95^{\circ} \mathrm{C}$ for $15 \mathrm{~s}$ and $60^{\circ} \mathrm{C}$ for $60 \mathrm{~s}$, and then incubation at $72^{\circ} \mathrm{C}$ for $10 \mathrm{~min}$ followed by real-time melt analysis to verify product specificity. Hprt1 was used as an internal control for normalization using the Ct method (Livak and Schmittgen, 2001). Oligonucleotide primer information is available on request.

RNA-seq. RNA libraries were produced and adaptor ligated; pairedend reads were sequenced on an Illumina HiSeq-2500 following library quality control. Quality control on raw reads was performed with FastQC (version 0.10.1) and adaptor trimming and removal of trimmed reads shorter than 20 bp was performed with Trimmomatic (Bolger et al., 2014). Trimmed reads were mapped to the UCSC Rattus norvegicus rn6 genome (version 0.13.0). Samtools (version 0.1.19-96b5f2294a) were used to sort SAM files and de-duplicate reads. Bowtie2 was used to align reads (Langmead and Salzberg, 2012). The subread featureCounts function was used to obtain counts. EdgeR was used for normalization and calculation of fold changes (Robinson et al., 2010). Gene ontology (GO) analyses were performed using Cytoscape (Shannon et al., 2003) with the BinGO plugin (Maere et al., 2005) and Gene set enrichment analysis (GSEA) (Subramanian et al., 2005).
Pyrosequencing of bisulfite-converted DNA. Bisulfite conversion of DNA was performed using the Qiagen EpiTect Bisulfite Kit. The NF- $\kappa$ B1 promoter primers were custom designed and then ordered through Integrated DNA Technologies. Amplification was performed using the Pyromark PCR kit. Pyrosequencing was performed using the Qiagen Pyromark Q24 system.

ChIP. ChIP analysis was performed as follows. First, $200 \mathrm{mg}$ of frozen tissue was homogenized, resuspended in PBS, and cross-linked in 1\% formaldehyde for $10 \mathrm{~min}$ at room temperature. The tissue pellet was resuspended in nuclei swelling buffer containing protease inhibitor. The separated nuclei were lysed in SDS lysis buffer containing protease inhibitors. The resulting chromatin was sonicated (Diagenode) at $4^{\circ} \mathrm{C}$ with 10 bursts for $30 \mathrm{~s}$, with a $30 \mathrm{~s}$ cooling interval between each burst. The average length of sonicated chromatin was determined by resolving it on a $1.2 \%$ agarose gel and was found to be $\sim 300-800 \mathrm{bp}$. The sample was then centrifuged at $13,000 \mathrm{rpm}$ for $10 \mathrm{~min}$ at $4^{\circ} \mathrm{C}$ to remove cell debris from the chromatin lysate. One milliliter of sheared chromatin was diluted in $10 \mathrm{ml}$ of ChIP dilution buffer. Ten percent of the diluted lysate was subsequently incubated overnight with $2 \mu \mathrm{g}$ of primary antibody/ magnetic bead solution at $4^{\circ} \mathrm{C}$. Normal rabbit IgG antibody was used as the negative control to demonstrate nonspecific binding. Antibodies were considered negative for binding if the resulting value was equal to or less than the IgG value (ratio to input). The supernatant of normal rabbit IgG was saved as the input control for PCR after clean-up. Antibody/ protein/DNA complexes were eluted from beads and then supernatants were incubated at $65^{\circ} \mathrm{C}$ for $4-5 \mathrm{~h}$ after the addition of $20 \mu \mathrm{l}$ of $5 \mathrm{~mol} / \mathrm{L}$ $\mathrm{NaCl}$ and $1 \mu \mathrm{g}$ of RNase A to reverse the formaldehyde cross-linking and to release the DNA fragments. Samples were treated with proteinase $\mathrm{K}$ at $37^{\circ} \mathrm{C}$ for $1 \mathrm{~h}$ to remove any protein and DNA was purified with a QiaPrep miniprep kit (Qiagen). Each real-time PCR used 5\% of immunoprecipitated DNA. Histone binding was expressed as the ratio to the input. Standards and the samples were simultaneously amplified in a $20 \mu \mathrm{l}$ reaction volume with primers designed to amplify genomic sequences at the promoter region of ARC.

Transcript/behavior correlation analysis. Correlation analysis was performed in MATLAB using Pearson correlation (corrcoef). For rats that performed the behavioral task (cohort 3 ), all transcripts changed by VNS with $p<0.1$ were included in the analysis. For hippocampal tissues, the RNA-seq dataset was used. Overall RNA-seq correlation significance was assessed by calculating the number of transcripts that correlated with a set of randomly drawn behavioral scores (uniform distribution with the same min and max as the true behavioral scores, $N=1000$ iterations). Morpheus was used to generate the correlation heat map (https://software.broadinstitute.org/morpheus). For cortex, qRT-PCR data were used to examine behavioral correlation because the cortical RNA-seq was performed on rats not subjected to the behavioral task. RNA-seq data were used to examine genes that correlated with ARC in the cortex.

Experimental design and statistical analysis. Details of experimental design are presented in the first two Results sections. Animal numbers were calculated based on parameters required to adequately power previous rodent studies. Data analysis was performed in R or MATLAB (version R2017; The MathWorks) and graphed using edgeR, Prism for Windows (version 6.00; GraphPad Software), or MATLAB. Normality was formally tested and verified where appropriate. Statistical significance was designated at $p<0.05$ for all analyses. Statistical significance was measured using two-sided unpaired $t$ tests. Adjusted $p$-values were calculated using ANOVA multiple comparisons. FDRs were calculated using Benjamini and Hochberg false discovery rate correction. For correlations, data were analyzed using Pearson correlation coefficients in MATLAB.

\section{Results}

Our study investigated whether epigenetic alterations underlie VNS enhancement of plasticity and cognition. The first set of experiments confirmed that 30 min of VNS during object familiarization can be used to enhance next-day novel object prefer- 

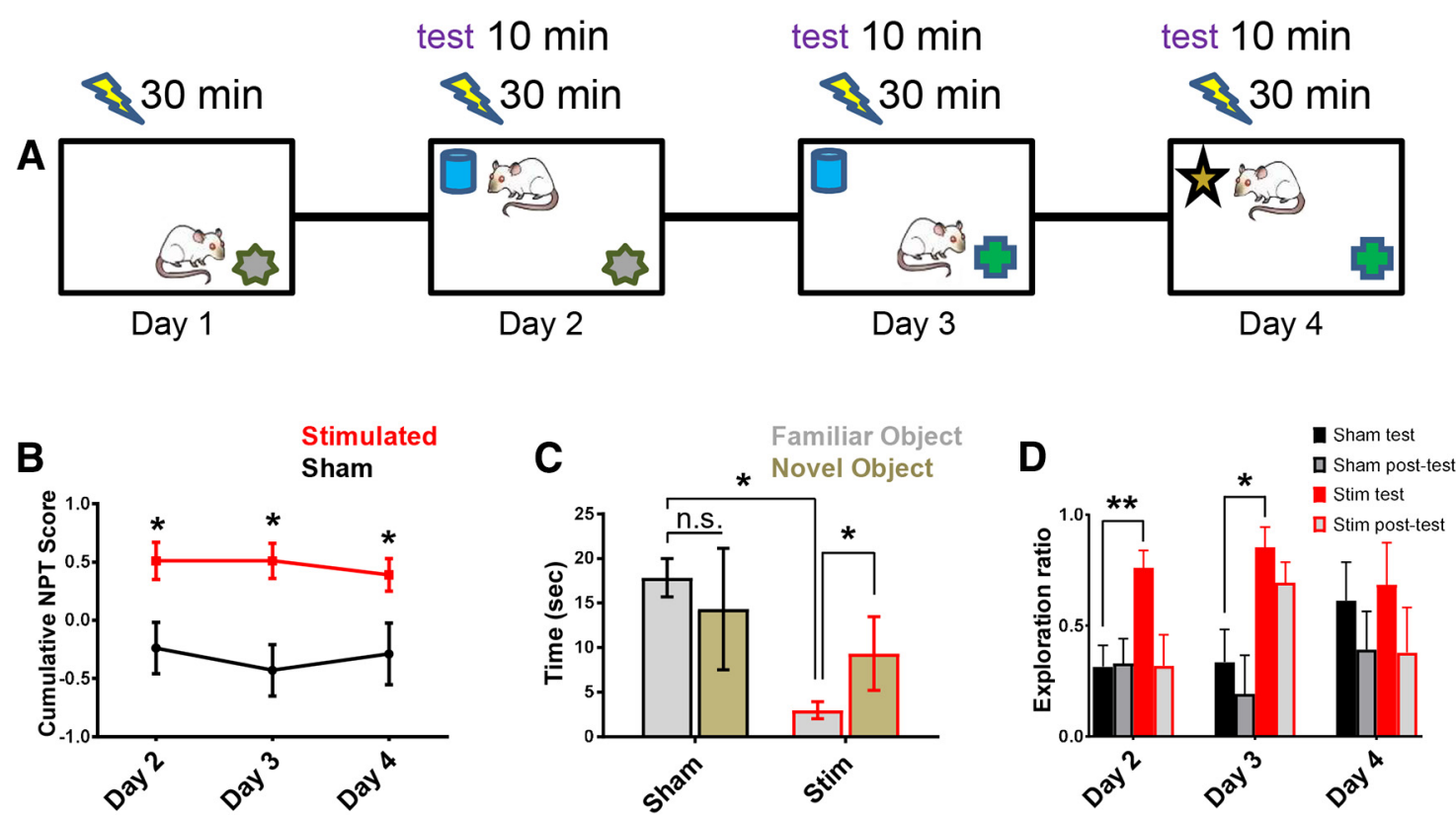

Figure 1. VNS enhances preference for novel objects. $A, 0$ day 1 , rats were introduced to the first object while receiving 30 min of stimulation. On day 2 and following, the object introduced on the previous day (familiar object) was placed in the same location and a novel object was introduced. Rats were observed for a 10 min test period while interacting with the two objects and then stimulated for $30 \mathrm{~min} . \boldsymbol{B}$, Rats receiving VNS displayed increased novel object preference compared with sham stimulated rats $\left(\Delta_{\text {novel_object_preference }}=0.7\right)$. C, VNS rats spent more time interacting with the novel objects than the familiar objects (ratio $=3.2$ ). Sham rats spent more total time interacting with the objects than VNS rats. The average time that shams spent interacting with the novel object was not significantly different from the average time spent with the familiar object. $D$, VNS rats, but not shams, explored the novel object more than the familiar object during all three 10 min test periods (exploration ratio $>0.5$ ). Novel object preference typically subsided during the 30 min stimulation period following the test period. $N=12$ rats, $n=5$ sham and $n=7$ stimulated, ${ }^{*} p \leq 0.05,{ }^{* *} p \leq 0.01$. Error bars indicate SEM.

ence in rats. The second set of experiments demonstrated that 4 consecutive days of 30 min VNS induces widespread epigenetic changes in the cortex, hippocampus, and blood, including altered epigenetic modulation of stress-response signaling and cortical plasticity genes.

\section{VNS enhances preference for novel objects}

We applied electrical stimulation 30 min per day through implanted cuff electrodes or sham cuffs on the left cervical vagus nerve in Sprague Dawley rats (see Materials and Methods). Stimulation parameters $(30 \mathrm{~Hz}, 0.8 \mathrm{~mA}, 100 \mu$ s biphasic pulses) were similar to the parameters used in previous rat and human VNS studies (Clark et al., 1999; Engineer et al., 2011). Rats were exposed to the first object on day 1 during VNS (Fig. 1A). On days $2-4$, the familiar object from the previous day (o1) was placed in its original location and a second object (o2) was placed in the opposite corner of the open field. Rats were tested for $10 \mathrm{~min}$ to determine whether they distinguished the novel object (o2) from the familiar object (o1). Cognitive enhancement was assessed by calculating the difference in the amount of time spent interacting with the novel object $\left(t_{\mathrm{o} 2}\right)$ compared with the familiar object $\left(t_{\mathrm{o} 1}\right)$, expressed as a fraction of the total time spent interacting with either object (novel object preference $\left.=\frac{t_{o 2}-t_{o 1}}{t_{o 2}+t_{o 1}}\right)$ (Haettig et al., 2011). Following testing, rats again received 30 min of VNS. During the test periods, rats that had received VNS demonstrated significantly increased novel object preference $\left(\Delta_{\text {novel_object_preference }}=0.7, p=0.02, n=12\right.$; Fig. $\left.1 B\right)$. VNS rats also spent less time interacting with the familiar object before interacting with the novel object, observed in previous studies to be an indicator of enhanced learning $\left(t_{\mathrm{o} 1 \mathrm{stim}} / t_{\mathrm{olsham}}=0.2, p=\right.$ 0.0003, $n=12$; Fig. 1C) (Broadbent et al., 2010; Gaskin et al., 2010). Compared with VNS rats, sham rats spent more time over- all interacting with the objects. This prolonged interaction time has been interpreted as an indication of slower learning in previous studies that allowed multiple familiarization days before novel object introduction (Gaskin et al., 2010; Antunes and Biala, 2012). Additionally, because the sham rats interacted with the novel object for a similar amount of time as the VNS rats (Fig. $1 C)$, it is unlikely that anxiety played a role in the sham rats' lack of preference for the novel object. Finally, it is important to note that, despite an average negative novel object preference measure in sham rats, the overall difference in the amount of time the sham rats spent interacting with the novel objects $\left(t_{\mathrm{o} 2}\right)$ versus the familiar objects $\left(t_{\mathrm{o} 1}\right)$ during the $10 \mathrm{~min}$ test phase was not statistically significant $\left(t_{\mathrm{o} 2 \mathrm{sham}} / t_{\mathrm{o} 1 \text { sham }}=0.7, p=0.45, n=5\right)$.

As observed in previous studies (Mumby et al., 2002), for each trial (day), novel object preference was generally diminished for both sham and stimulated rats after several minutes of exploration (Fig. 1D). Interestingly, although the cumulative novel object preference was significant over all $3 \mathrm{~d}$ (Fig. $1 B$ ), the daily exploration ratio, determined by $\frac{t_{o 2}}{t_{o 2}+t_{o 1}}$ (Mumby et al., 2002) for VNS rats compared with shams, was significantly increased on days 2 and 3 ( $p=0.01$ and $p=0.05$, respectively), but not on day $4(p=0.62)$, perhaps due to increased novelty preference with days of participation for the sham rats (Fig. $1 D$ and see Discussion).

Four $30 \mathrm{~min}$ sessions of VNS changed expression of plasticity genes in the cortex, hippocampus, and blood

For our second set of experiments, we extracted brain and blood samples from three cohorts of rats: cohort 1 received a single 30 min session of VNS $(N=15, n=8 \mathrm{VNS}$ and $n=7$ sham $)$, cohort 2 received 4 consecutive days of VNS with no task $(N=8$ rats, $n=$ $4 \mathrm{VNS}$ and $n=4$ sham), and cohort 3 received 4 consecutive days 

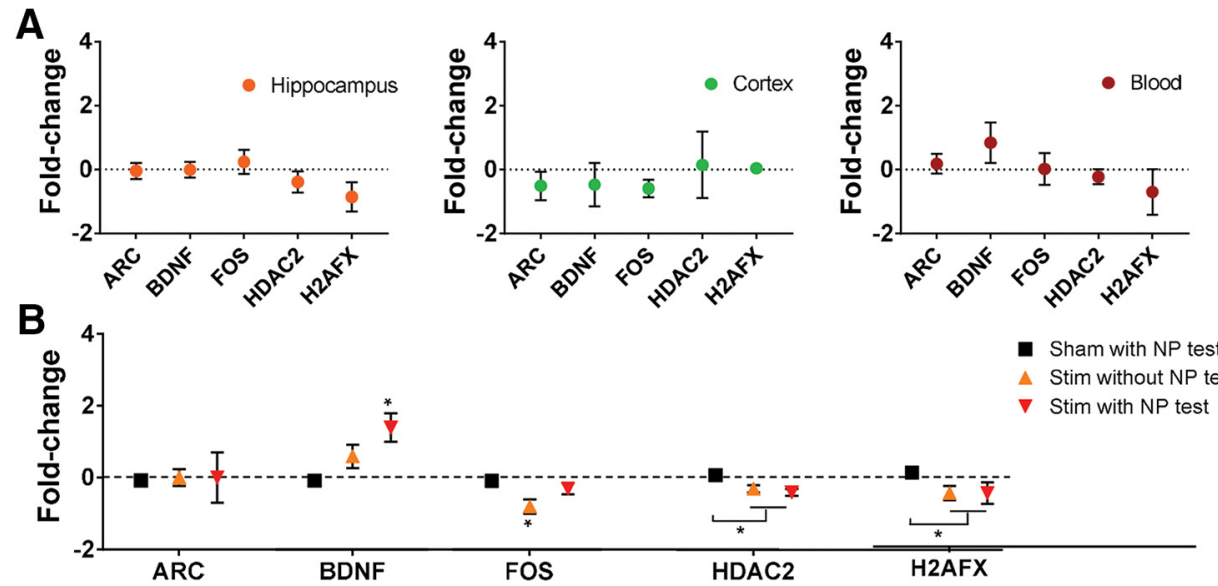

\section{Day 1 RT-qPCR}

Day 4 RT-qPCR Hippocampus
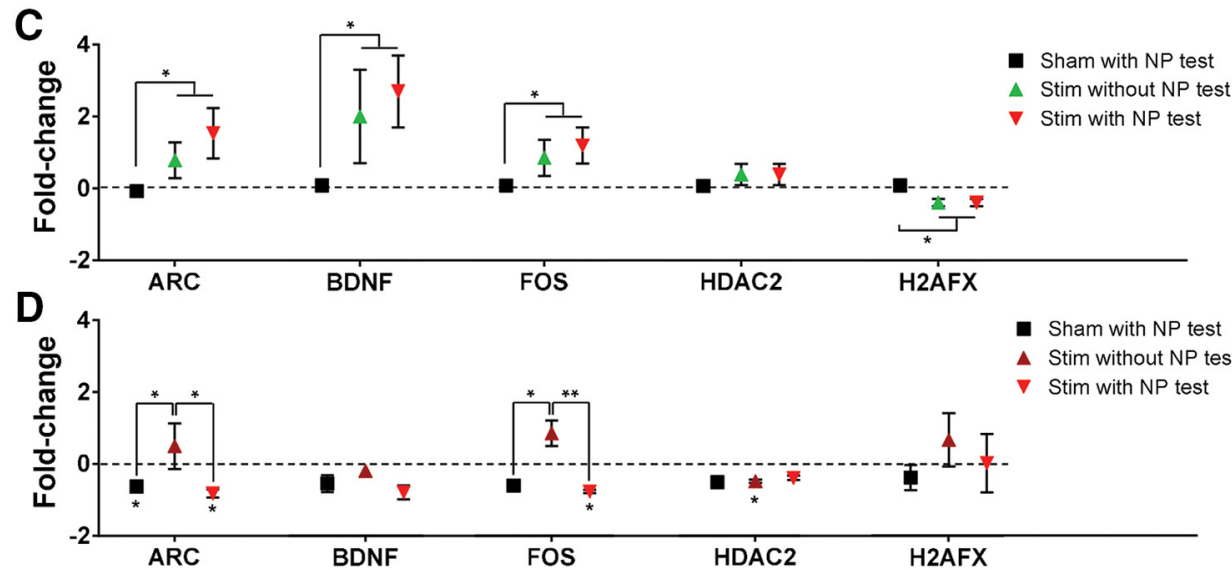

\section{Day 4 RT-qPCR Cortex}

\section{Day 4 RT-qPCR} Blood

Figure 2. Thirty minutes of VNS delivered on 4 consecutive days modulates transcription of a panel of learning- and memory-associated genes. $A$, Transcript quantities in the hippocampus, cortex, and blood did not change significantly after a single 30 min VNS treatment. B, C, Plasticity-related transcripts were significantly changed by VNS in the cortex and hippocampus after $4 \mathrm{~d}$ of stimulation (stim). Transcripts from rats participating in the NP behavioral task (downward pointing triangle) changed in the same direction as transcripts from rats that received stimulation alone (upward pointing triangle). D, In the blood, IEG transcripts (ARC and FOS) from rats that received stimulation alone were increased, whereas transcripts from rats that participated in the NP task were reduced. $\boldsymbol{A}$, Results from cohort 1 (single day of stim). For $\boldsymbol{B}-\boldsymbol{D}$, results from cohort 2 are indicated by the dashed line (sham) and the upward pointing triangle (stim), whereas results from cohort 3 are indicated by the black squares (sham) and the downward pointing triangle (stim). Asterisks without pair-bars reflect significance relative to the dashed line (sham without NP test). ${ }^{*} p<0.05$, ${ }^{* *} p<0.01$, unpaired $t$ test. Error bars indicate SEM.

of VNS during the 30 min object familiarization period $(N=12$ rats, $n=7$ VNS and $n=5$ sham; Fig. 1 ). Samples were extracted within 30 min of the last session of VNS for each rat. We did not find evidence of changes in plasticity gene expression in cohort 1 ( single session of VNS with no consolidation; Fig. $2 A$ ). However, in cohorts 2 and 3, qRT-PCR (Fig. 2B-D) and Western blot (data not shown) analysis revealed multiple changes in expression of plasticity-related genes in cortex, hippocampus, and blood. Subsequent RNA-seq revealed widespread transcriptional changes in epigenetic modulators, stress-response signaling, and plasticity genes in both the cortex and hippocampus (see Figs. 3, 5). Specific epigenetic investigation revealed DNA methylation changes and learning-related histone modifications.

Initially, we examined a panel of genes known to be involved in learning and memory, including the IEGs ARC and FOS, a neurotrophic factor (BDNF), and epigenetic regulators [histone deacetylase 2 (HDAC2) and H2AFX, the gene that encodes histone $2 \mathrm{~A}$ variant $\mathrm{X}(\mathrm{H} 2 \mathrm{~A} . \mathrm{X})$ ]. Results from this initial panel were used to guide further assays and analysis.

We found no significant changes in any of the panel genes after a single $30 \mathrm{~min}$ session of VNS (cohort $1, N=15, n=8 \mathrm{VNS}$ and $n=7$ sham; Fig. $2 A$ ). However, after 4 consecutive days of 30 min stimulation, we found significantly changed transcription (Fig. 2B-D). The differential transcription profiles varied be- tween the three locations sampled. In the hippocampus, we found that transcripts for each gene changed in the same direction in VNS rats (increased or decreased) regardless of whether the NP test was performed (Fig. $2 B$, cohort 2 without the NP test, $N=8$ rats, $n=4 \mathrm{VNS}$ and $n=4$ sham, orange upward-pointing arrow; cohort 3 with the NP test, $N=12$ rats, $n=7 \mathrm{VNS}$ and $n=$ 5 sham, red downward-pointing arrow). This was also true in the cortex, where the largest transcriptional changes occurred in $\mathrm{BDNF}$ (fold change $=2.7, p=0.05$ ), ARC (fold change $=1.5$, $p=0.04$ ), and FOS (fold change $=1.2, p=0.05$ ) (Fig. $2 C$ ).

HDAC2 transcripts were decreased in both hippocampus and blood (Fig. $2 B, D$ ). H2AFX (associated with DNA double-strand breaks) transcripts were decreased in the hippocampus and cortex (Fig. $2 B, C$ ).

\section{IEGs ARC and FOS transcript quantities in the blood depend} on whether rats performed the NP task

Interestingly, in the blood, the direction of the transcriptional changes for the IEGs depended on whether the NP task was performed rather than on whether the rat received stimulation (Fig. $2 D$ and see Discussion). Rats that received VNS without the NP task had increased ARC and FOS, whereas VNS rats that performed the task had reduced ARC (fold change $=-0.8, p=$ 0.0004 ) and FOS (fold change $=-0.8, p=0.018$ ). Note that this 

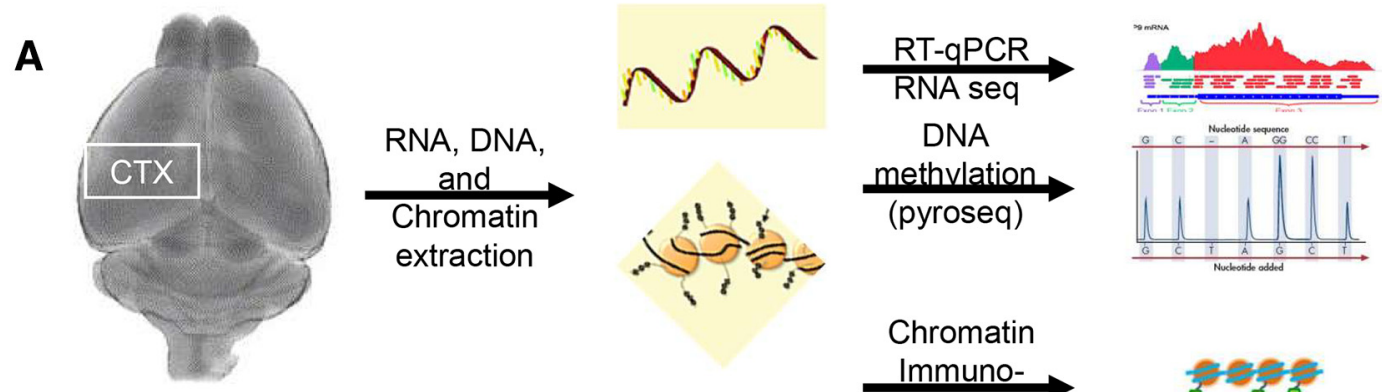

(pyroseq)
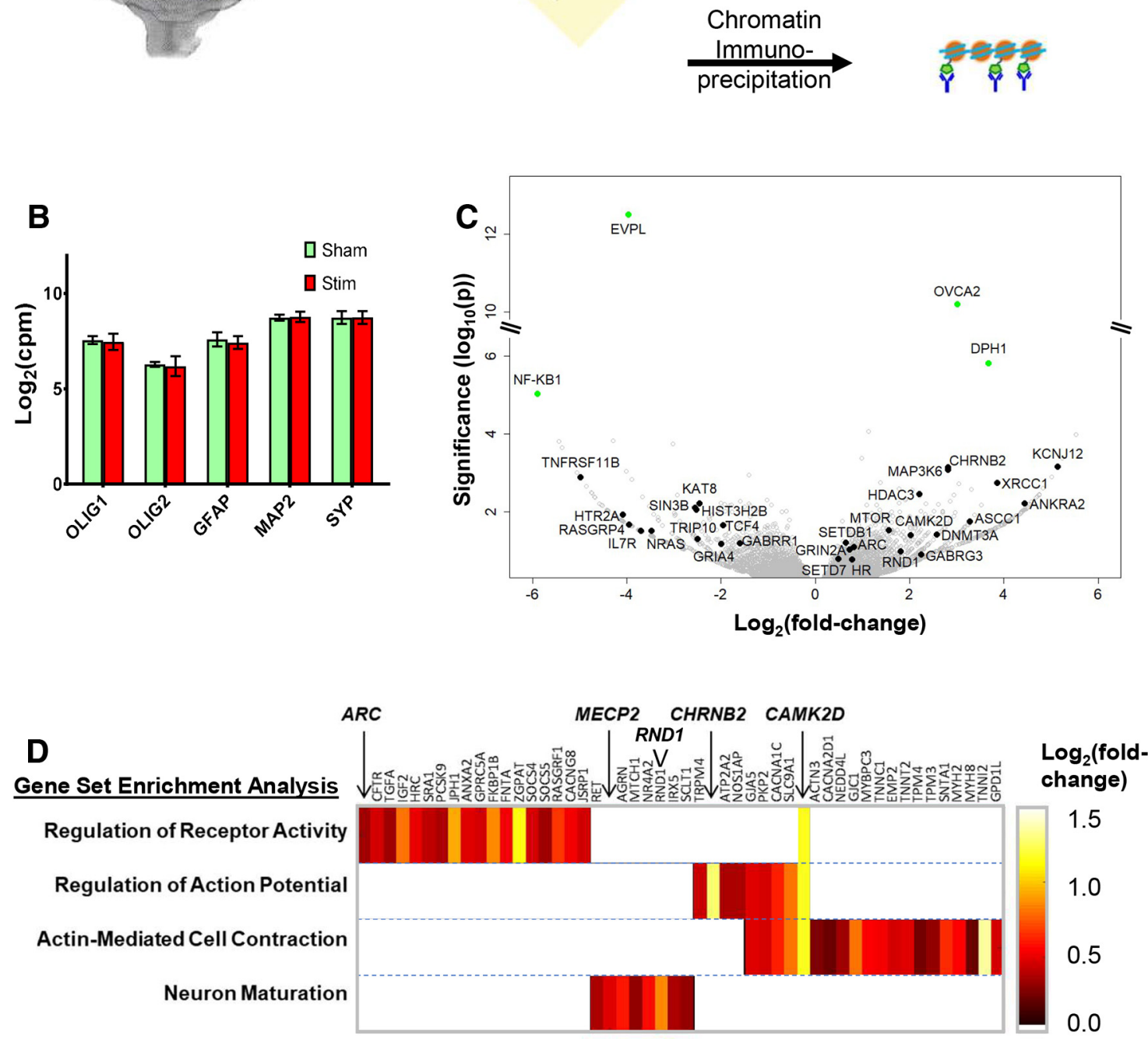

Figure 3. VNS alters cortical transcription. $\boldsymbol{A}$, RNA-seq, DNA methylation, and ChIP data analysis pipeline. $\boldsymbol{B}$, Cortical cell identity transcripts revealed evidence of both glial (OLIG1, 0 LIG2, GFAP) and neuronal (MAP2, SYP) cells. However, no significant differences were found between the sham and stimulated cell identity transcripts. Error bars indicate SEM. C, Differentially transcribed cortical genes in VNS versus sham-stimulated rats $(p<0.01)$. Genes annotated with text are significantly changed either individually (FDR $<0.25$, filled blue circles) or as part of a $\mathrm{G} 0$ group (adjusted $p<0.05$, filled black circles). D, Synaptic plasticity G0 categories upregulated by VNS (GSEA biological process categories, $p<0.01$ ).

NP task-associated reduction in blood transcripts was also significant for ARC in the sham rats (black square, fold change $=-0.6$, $p=0.013)$.

Based on this initial evidence that VNS changes transcription of IEGs and epigenetic regulators, we chose to further probe the VNS-induced transcriptional changes. We analyzed RNA-seq results from cortical and hippocampal tissues (see Figs. 3, 5) extracted from VNS and sham rats that received $30 \mathrm{~min}$ of stimulation on 4 consecutive days. Although further analysis of the trunk blood would have been beneficial, we did not pursue blood RNA-seq because initial sample quality checks did not meet the stringent thresholds recommended by our RNA-seq process.

We verified the cell populations used for RNA-seq were balanced between the sham and stimulated rat samples by quantify- ing cell identity transcripts. The RNA-seq mRNA counts for a standard panel of cell identity transcripts showed no significant differences between the samples from sham and stimulated rats for either the cortex (Fig. 3B) or the hippocampus (Fig. 5C).

VNS without a task induces widespread changes in cortical transcription

Because VNS caused significant cortical plasticity even in rats that did not perform a behavioral task (Fig. 2C), we examined the overall "preparatory" cortical plasticity changes induced by VNS alone (Fig. 3, cohort 2). In all, 427 cortical genes were differentially transcribed (Fig. $3 C, \| \log _{2}$ (fold change) $\|(\log \mathrm{FC})>1, p<$ $0.05, N=8$ rats, $n=4 \mathrm{VNS}$ and $n=4$ sham), with a majority indicating increased transcription (55\%, 233 of 427). VNS 
strongly increased transcription of plasticity genes such as CAMK2D $(\log \mathrm{FC}=$ $2.0 p=0.04)$, translation elongation factor modifier DPH1 $(\log \mathrm{FC}=3.7, p=$ $1.5 \times 10^{-6}$ ), and ovarian tumor suppressor candidate 2 OVCA2 $(\operatorname{logFC}=3.0, p=$ $\left.7.0 \times 10^{-11}\right)$. Nicotinic acetylcholine receptor subunit $\beta$-2 (CHRNB2) was also increased $(\log \mathrm{FC}=2.8, p=0.0007)$, likely due to VNS upregulation of cholinergic signaling. MAP3K6, a MAP kinase important for vascular endothelial growth signaling, was also increased $(\log \mathrm{FC}=$ $2.8, p=0.0008)$. The most decreased cortical transcripts were stress-response signaling transcription factor subunit NF$\kappa \mathrm{B} 1\left(\log \mathrm{FC}=-5.9, p=9.6 \times 10^{-6}\right)$, EVPL $(\log F C=-4.0, p=3.5 \mathrm{E}-13)$, and cytokine receptor TNFRSF11B, also known as osteoprotegerin ( $\mathrm{OPG}, \log \mathrm{FC}=$ $-5.0, p=0.01)$.

VNS also induced transcriptional changes in many epigenetic modulators important for learning and memory, such as HDAC3 $(\log \mathrm{FC}=2.2, p=0.0035)$ and de novo DNA methyltransferase DNMT3A $(\log \mathrm{FC}=2.6, p=$ 0.015). Transcription of KAT8 (MYST1), a histone modifier that acetylates lysine 16 on $\mathrm{H} 4$ (H4K16ac, associated with open chromatin), decreased $(\log \mathrm{FC}=-2.5, p=0.006)$ and correlated with reduced transcription of RASGRP4 [Pearson correlation coefficient $(\rho)=0.98, p=2.8 \mathrm{E}-5]$ and cytokine receptor TNFRSF11B $(\rho=0.85, p=0.007)$. HIST3H2B, typically increased in the presence of DNA DSBs, decreased as well $(\operatorname{logFC}=-2.6, p=$ $0.009)$.

GO analysis revealed increased transcription in several important plasticity categories (Fig. 3D, GSEA Biological Process gene set Version c5.bp.v6.1.symbols.gmt; Subramanian et al., 2005). These GO categories included genes known to be critical for learning and memory such as ARC, MECP2 (methyl CpG binding protein 2), CAMK2D, and CHRNB2. The GO category "regulation of myelination" was also upregulated in part due to increased transcription of HDAC3 and MTOR $(\operatorname{logFC}=1.6, p=$ 0.03 ), a serine/threonine protein kinase that regulates myelination in conjunction with cell growth, cell survival, protein synthesis, autophagy, and transcription. GO categories specifically related to epigenetic mechanisms were also increased, including "Histone $\mathrm{H} 3$ deacetylation."

Other studies have addressed the cholinergic effects of VNS. Therefore, we chose to focus on genes newly linked to VNS by our transcriptome analysis.

Stress-response signaling, neuronal plasticity, and epigenetic regulation transcripts correlate with one another

To further probe the signaling related to the reduced stress-response transcripts, we examined correlations between NF- $\kappa \mathrm{B} 1$ and other genes in the cortical RNA-seq data. Ninety-five genes correlated significantly with NF- $\kappa \mathrm{B} 1$ (Pearson correlation, $\operatorname{abs}(\rho)>0.8, p<0.01$, see methods). Positively correlated genes included NRAS, IL7R, and AMPA and $\mathrm{GABA}_{\mathrm{A}}$ receptor subunits (GRIA4, GABRR1).

Because neural plasticity gene ARC was significantly increased in the cortex of rats from both cohort 2 and cohort 3, we also investigated the 124 RNA-seq transcripts that correlated with
ARC $(\operatorname{abs}(\rho)>0.8, p<0.01)$. Positively correlated genes included receptor subunits (GABRG3, GRIN2D) and multiple epigenetic regulators. ARC transcription negatively correlated with transcription of serotonin receptor 5A (HTR5A), and matrix metalloproteinase-17 (MMP17). 5- $\mathrm{HT}_{5 \mathrm{~A}}$ negatively influences cAMP ( $\mathrm{G}_{\mathrm{i}}$ protein-coupled $)$ and has been implicated in psychiatric conditions such as schizophrenia. Increased MMPs are found to associate with disease processes such as arthritis and metastasis due to their degradation of the extracellular matrix, so the decrease provides supporting evidence for a VNS therapeutic effect on arthritis (Bassi et al., 2017).

Transcription of many epigenetic regulators correlated with cortical ARC transcription, including SETD7 (H3K4 histone methyltransferase involved in control of cell proliferation, differentiation, and ER stress), SETDB1 (histone methyltransferase involved in $\mathrm{H} 3 \mathrm{~K} 9$ methylation and $\mathrm{H} 3 \mathrm{~K} 14$ acetylation), ANKRA2 (involved in HDAC Class II signaling), DNMT3A, and HDAC3 (class I HDAC). To identify potential interactions between transcripts that correlated with NF- $\kappa \mathrm{B} 1, \mathrm{ARC}$, and the ARCcorrelating epigenetic regulators, we mapped the network of correlations between four transcript classes: plasticity genes, epigenetic regulators, stress-response signaling, and immune receptors (Fig. 4). Epigenetic regulators that correlated with ARC transcription heavily regulated plasticity genes that were significantly changed by VNS (Fig. $4 A, B$ ). We also found that TNFRSF11B (OPG) correlated with genes from all of the other classes (Fig. 4D).

\section{VNS induces widespread changes in hippocampal} transcription when paired with a novelty preference task To identify hippocampal changes induced by VNS, we analyzed RNA-seq data from rat samples extracted immediately after stimulation on day 4 of the NP behavioral task $(N=7$ highest quality hippocampal RNA samples from cohort 3: $n=4$ VNS, $n=3$ sham). Hippocampal RNA-seq analysis identified 524 genes with significant transcription changes; $61 \%$ were decreased $(N=7$ rats, $n=4$ VNS and $n=3$ sham; Fig. 5). This result was supported by ChIP results indicating reduced levels of hippocampal 
A

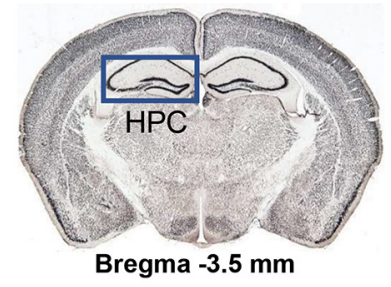

B

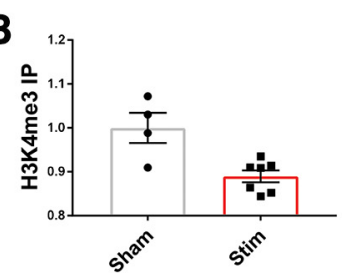

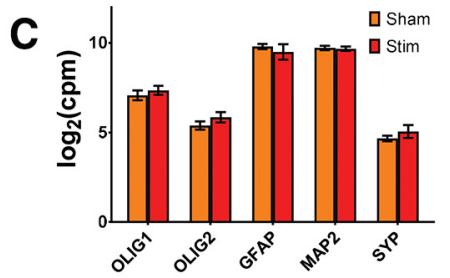

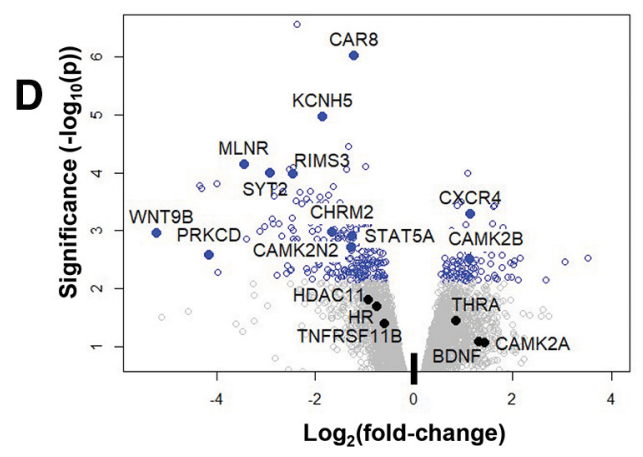

$\mathbf{F}$

Gene Set Enrichment Analysis $\log _{2}$ (fold-change) NEURON RECOGNITION NEGATIVE REGULATION OF ER STRESS CHAPERONE MEDIATED PROTEIN FOLDING DNA TEMPLATED TRANSCRIPTION ELONGATION NEGATIVE REGULATION OF STAT CASCADE POSITIVE REGULATION OF DNA BINDING

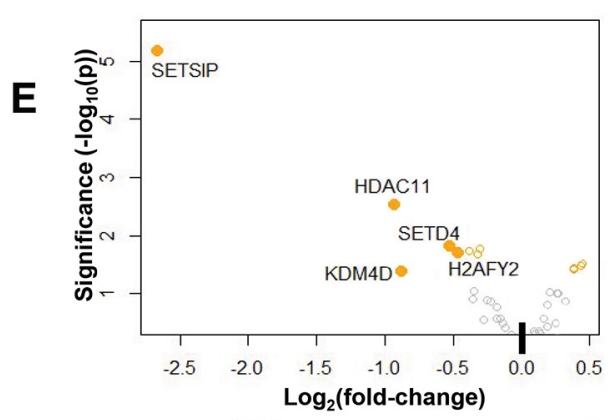

G

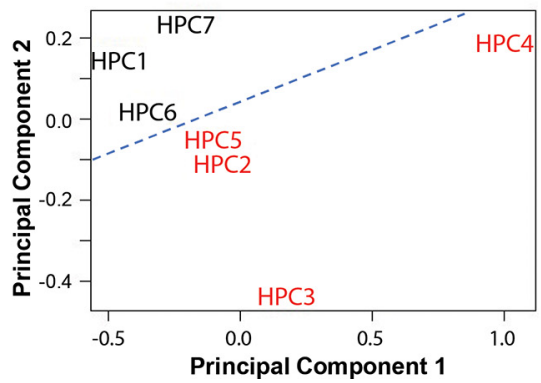

Figure 5. VNS alters hippocampal transcription. $\boldsymbol{A}$, Location of hippocampal sample shown on a coronal rat brain section. $\boldsymbol{B}$, H3K4me3, an epigenetic marker found in active promoters, was decreased in the hippocampus, suggesting reduced transcription. $\boldsymbol{C}$, As in the cortex, cell identity transcripts revealed the presence of both glial and neuronal cells. Although the profile differed from that of cortical transcripts (increased GFAP and reduced SYP), no significant differences were found between the sham and stimulated cell identity transcripts. Error bars indicate SEM. $\boldsymbol{D}$, Differentially transcribed genes in VNS versus sham rats $(p<0.01)$. Genes annotated with text are significantly changed (FDR $<0.25$, filled blue circles) or as part of a G0 set (adjusted $p<0.05$, filled black circle). $\boldsymbol{E}$, Differentially transcribed epigenetic modulation genes in VNS versus sham rats $(p<0.01)$. Genes annotated with text are significantly changed $(p<0.01$, FDR $<0.25)$. $\boldsymbol{F}$, Representative significantly increased G0 categories from GSEA Biological Processes $(p<0.01)$. G, Principal component analysis of hippocampal epigenetic transcript data enables separation of the sham (black) and VNS (red) rats.

H3K4me3, a histone methylation mark associated with increased transcription (Fig. 5B). VNS strongly decreased transcription of WNT9B $(\operatorname{logFC}=-5.2, p=0.001)$, known to be important for calcium signaling, cell proliferation and differentiation, PRKCD (protein kinase $\mathrm{C}$ delta, $\log \mathrm{FC}=-4.1, p=0.003$ ), a cell cycle regulator that can play a role in activation of $\mathrm{NF}-\kappa \mathrm{B}$ pathways, and motilin receptor, MLNR $(\log \mathrm{FC}=-3.4, p=7.0 \mathrm{E}-5)$, important for both brain and gut motility. Increased transcripts included ubiquitin-associated chemokine receptor CXCR4 $(\log \mathrm{FC}=1.1, p=0.0005)$ and CAMK2B $(\log \mathrm{FC}=1.1, p=$ 0.003 ) (Fig. 5D).

GO analysis revealed further diverse effects in the hippocampus (Fig. $5 F$ ), including changes in transcripts involved in increased chaperone-mediated protein folding, negative regulation of ER stress, negative regulation of STAT signaling, increased transcription elongation, and reduced transmembrane transport.

\section{VNS changes hippocampal calcium signaling}

Many significantly differentially transcribed genes in the hippocampus were related to calcium signaling, including CAMK2B, CAMK2N2, SYT2, and Car8 (Fig. 5D). CAMK2 $\left(\mathrm{Ca}^{2+} /\right.$ calmodulin-dependent protein kinase 2$)$ is involved in many signaling cascades, including stress response, and is an important mediator of learning and memory (Lisman et al., 2002). CAMK2B encodes the $\beta$ chain of CAMK2 (one of four CAMK2 subunits), whereas CAMK2N2 inhibits CAMK2. The observed increase in CAMK2B and decrease in CAMK2N2 $(\operatorname{logFC}=-1.3$, $p=0.002)$ suggests that VNS promotes net CAMK2 activity. CAMK2B correlated with increased BDNF $(\rho=0.76, p=0.05)$. SYT2 (synaptotagmin 2) is a synaptic vesicle membrane protein and calcium sensor $(\log \mathrm{FC}=-2.9, p=0.0001)$. Finally, Car8 (carbonic anhydrase $8, \log \mathrm{FC}=-1.2, p=8.8 \times 10^{-7}$ ) inhibits a receptor that promotes intracellular calcium release. Previous studies have shown that histone modifiers can play an important role in calcium regulation through Wnt and other signaling pathways (Takada et al., 2009). In our study, we found multiple threeway correlations among novel object recognition, calcium signaling transcripts, and epigenetic regulation transcripts (Fig. 6).

\section{VNS alters the hippocampal epigenetic transcriptome}

RNA-seq data from hippocampal tissues was separable into VNS and sham-stimulated populations based solely on changes in epigenetic modulator transcription (Fig. $5 E, G$ ). The separation was enabled using only the first two principal components from a set composed of histone deacetylases, histone acetyltransferases, histone methyltransferases, histone demethylases, DNA methyltransferases, and ten-eleven translocation (TET) enzyme gene transcripts from the rattus norvegicus rn6 reference genome.

The most reduced hippocampal epigenetic transcript was SETSIP $(\operatorname{logFC}=-2.7, p=5.4 \mathrm{E}-06)$, a gene similar to SET that encodes a histone $\mathrm{H} 4$ acetylation (HAT) inhibitor. The encoded 

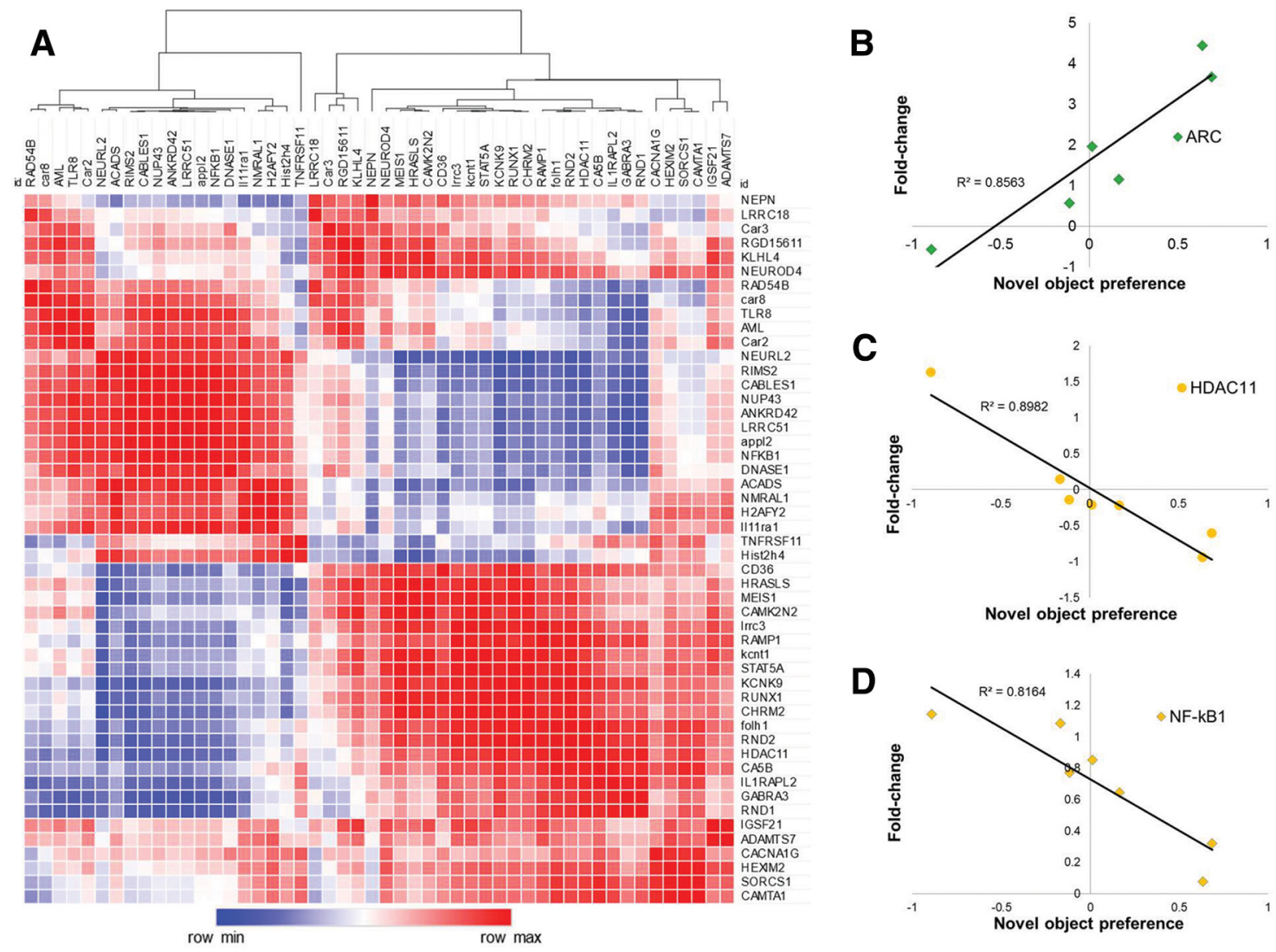

Figure 6. VNS-induced transcription correlates with novel object preference. $A$, Pearson correlation between 50 hippocampal transcripts that correlate with novel object preference (abs $(\rho)>$ 0.8). Note TNFRSF11 refers to TNFRSF11B. $B$, Cortex: IEG ARC transcription correlates with novel object preference $\left(R^{2}=0.8563\right.$, qRT-PCR). C, Hippocampus: Epigenetic regulator HDAC11 transcription is inversely correlated with novel object preference $\left(R^{2}=0.8982\right.$, RNA-seq). $D$, NF- $\kappa B 1$ transcription is inversely correlated with novel object preference $\left(R^{2}=0.8164\right.$, RNA-seq).

protein is part of a complex typically localized to the endoplasmic reticulum but also found in the nucleus. Decreased SETSIP correlated with reductions in TNFSF13B $(\rho=0.89, p=0.007)$ and TNFSF14 $(\rho=0.92, p=0.004)$. Other reduced transcripts included those for genes encoding HDAC11 (histone deacetylase enriched in output cells of the hippocampus CA1; Broide et al., 2007), histone H2A.Y2 (H2AFY2, a learning and memoryassociated histone variant; Barrero et al., 2013), SETD4 (lysine methyltransferase), and KDM4D (histone lysine (H3K9) demethylase). HDAC11 and H2AFY2 correlated with many hippocampal transcriptional changes $(\rho>0.8, p<0.01$; Fig. 6$)$. SETD4 correlated with NF- $\kappa$ B1 $(\rho=0.98, p=0.0002)$, IL17RC $(\rho=0.98, p=9.7 \mathrm{E}-5)$, RASGRP3 $(\rho=0.96, p=0.0006)$, and GABRB2 $(\rho=0.89, p=0.007)$. Reduced KDM4D correlated with reduced SYT2 $(\rho=0.91, p=0.004), \operatorname{KCNH} 5(\rho=0.91, p=$ $0.005), \operatorname{Car} 2(\rho=0.96, p=0.0008), \operatorname{CAMK} 2 \mathrm{~N} 2(\rho=0.93, p=$ $0.002)$, and GABRB2 $(\rho=0.94, p=0.002)$. Clearly, VNS alters the epigenetic transcription landscape associated with stress-response signaling and neuronal plasticity. Because our interest was in epigenetic changes related to cognition, we sought to identify significantly changed genes involved in the observed enhanced novel object preference.

\section{VNS-induced transcriptome alterations correlate with behavioral performance}

To identify genes likely to be directly involved in learning and memory, we examined correlations between behavior and transcription in the most significantly changed transcripts. Pearson correlation analysis revealed that transcription levels in several genes were correlated with one another and with performance on the NP behavioral task (Fig. 6). In particular, cortical ARC transcription was strongly positively correlated with NP test performance $(\rho=0.94, p=0.01$, qRT-PCR; Fig. $6 B)$.

In the hippocampus, transcription of 714 genes correlated with NP test performance $(\operatorname{abs}(\rho)>0.8, p<0.01$, RNA-seq). The most correlated gene was SORCS1 $(\rho=-0.98, p=7.9 \mathrm{E}-5)$, which encodes a protein important for vacuolar protein sorting. SORCS1 is genetically associated with diabetes and Alzheimer's amyloid- $\beta$ metabolism (Lane et al., 2010). Interestingly, NF- $\kappa$ B1 $(\rho=-0.90, p=0.005)$ and TNFRSF11B (OPG, $\rho=-0.95, p=$ 0.001 ), found to be significantly changed in the cortical RNA-seq, were included in the top hippocampal genes that correlated with behavioral performance. Many of the 714 genes correlated significantly with one another (Fig. 6A). Hierarchical clustering of the Pearson correlations revealed two major clusters, one that contained HDAC11, and another that contained H2AFY2 and NF$\kappa \mathrm{B} 1(\mathrm{H} 2 \mathrm{AFY} 2 \mid \mathrm{NF}-\kappa \mathrm{B} 1 \rho=0.92, p=0.0028)$.

Genes that clustered with HDAC11 included CAMK2N2 ( $\rho=$ $-0.89, p=0.007)$, STAT5A $(\rho=-0.94, p=0.001)$, folh1 $(\rho=$ $-0.97, p=0.0004)$, and parasympathetically linked muscarinic acetylcholine receptor, CHRM2 $(\rho=-0.91, p=0.005)$. The top upregulated gene was plectin (PLEC, a protein that bins intermediate filaments, actin microfilaments and microtubules and forms links between other cellular components, observed in astrocytic end feet abutting on blood vessels). Genes that clustered with NF- $\kappa \mathrm{B} 1$ and H2AFY2 included DNA damage checkpoint regulator, $\operatorname{RAD} 54 \mathrm{~B}(\rho=-0.88, p=0.009)$, Car8 $(\rho=-0.91$, $p=0.004)$, and TNFRSF11B (OPG). These correlations suggest 
A

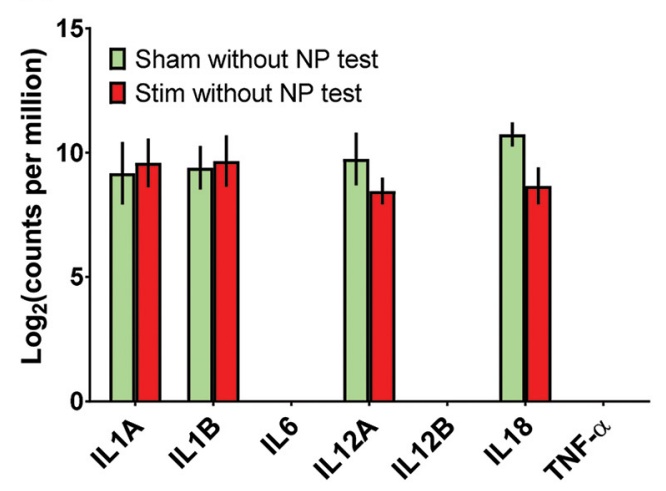

B

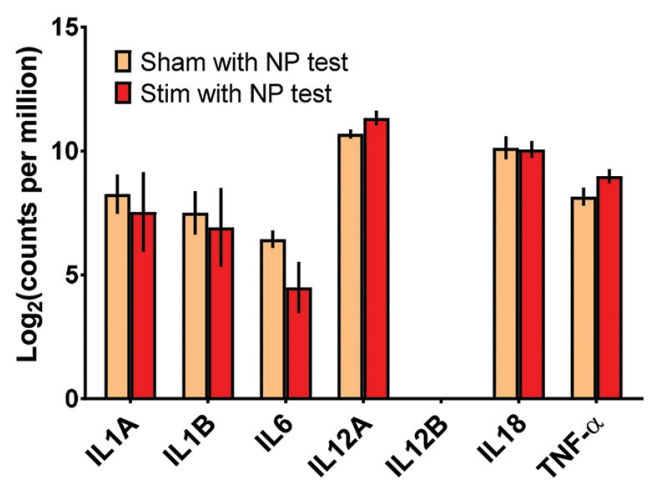

Figure 7. Proinflammatory cytokine transcripts are not significantly changed after $4 \mathrm{~d}$ of VNS. $A$, Cortical IL12A and IL 18 were reduced, but did not reach significance $(p=0.32$, and $p=0.06$, respectively, RNA-seq data, $n=8$ ). B, Hippocampal proinflammatory transcripts were not significantly changed (IL6 $p=0.18$, RNA-seq data, $n=7$ ). Error bars indicate SEM.

relationships among transcription of epigenetic regulators, neuronal plasticity, stress-response signaling, and other powerful signaling cascades that may be responsible for the enhanced cognition observed in the NP task.

The many correlated neuronal plasticity, stress-response signaling, and epigenetic regulation transcriptional changes in the VNS rats led us to investigate how these three categories of genes might interact. In particular, we were interested in the epigenetic regulation of stress-response signaling and neuronal plasticity genes important for VNS-enhanced learning and memory.

\section{VNS downregulates stress-response signaling without} significantly changing inflammatory cytokine transcripts As previously discussed, cortical NF- $\kappa \mathrm{B} 1$ transcripts were significantly reduced by VNS (see Figs. $3,8 A$ ). NF- $\kappa$ B1 was also decreased in the blood ( $\mathrm{qRT}-\mathrm{PCR}, \log \mathrm{FC}=-1.6, p=0.03$ ) and inversely correlated with novel object preference in the hippocampus (Fig. $6 D$ ). NF- $\kappa \mathrm{B}$ is a transcription factor known to be a master regulator of dynamic response mechanisms such as immune activation, cell proliferation, and neural plasticity. Our finding of reduced NF- $\kappa \mathrm{B} 1$ in VNS rats is congruent with previous studies showing decreased inflammatory signaling during VNS treatment (Borovikova et al., 2000; Tracey, 2002). Interestingly, our analysis found decreased NF- $\kappa \mathrm{B} 1$ without corresponding decreases in transcription of proinflammatory cytokines (Fig. 7, RNA-seq). Anti-inflammatory cytokine transcripts including IL10 and TGF- $\beta$ also failed to show significant increases. We hypothesized that VNS may downregulate stress response signaling through other known NF- $\kappa \mathrm{B}$ modulators such as hormones, histone modifiers, or DNA DSBs.

The correlation between NF- $\kappa$ B1 and TNFRSF11B (OPG, reduced in cortex and hippocampus) suggested a potential involvement of thyroid and/or parathyroid hormone-mediated effects in reduced stress-response signaling. Although we did not find significant changes in thyroid or parathyroid hormone expression, we found increased hippocampal thyroid hormone receptor $\alpha$ (THRA, $\log F C=1.1, p=0.01$ ) and reduced cortical thyroid hormone receptor interactor 10 (TRIP10, $\operatorname{logFC}=-2.5, p=$ $0.05)$. TRIP10 was also decreased in the hippocampus and correlated with NF- $\kappa \mathrm{B} 1(\rho=0.97, p=0.0002)$, along with epigenetic modifiers H2AFY2, SETD4, and KDMD4. Interestingly, examination of a set of thyroid hormone-responsive genes, FOS (Fig. $2 B, C$ ), STAT5A (Fig. 5D, Fig. 6), lysine demethylase HR, and TXNIP (Chatonnet et al., 2015; Kyono et al., 2016), revealed increased transcription of the genes in the cortex and decreased transcription in the hippocampus (see Discussion).

Given the known link between DNA DSBs and stress-response signaling (Volcic et al., 2012; Madabhushi et al., 2015) and our evidence suggesting involvement of VNS epigenetic modulation in both, we examined epigenetic regulation of NF- $\kappa \mathrm{B} 1$.

\section{VNS decreases DNA methylation at the NF- $\kappa \mathrm{B} 1$ promoter in both the cortex and hippocampus}

Because CpG methylation at gene promoters is known to regulate transcription, we used bisulfite conversion followed by pyrosequencing to examine DNA methylation in the NF- $\kappa \mathrm{B} 1$ promoter region in VNS and sham rats $(N=8, n=4$ sham and $n=4 \mathrm{VNS}$, cortex: cohort 2, hippocampus: cohort 3; Fig. 8). Although reduced gene body methylation can coincide with decreased transcription (Yang et al., 2014), gene promoter methylation is typically found to be increased with decreased transcription. Surprisingly, our results showed that NF- $\kappa \mathrm{B} 1$ promoter $\mathrm{CpG}$ methylation was reduced in rats that received VNS compared with those that received sham stimulation $(23 \%$ overall decrease in cortex, $p=0.01$, unpaired $t$ test; Fig. $8 B$ ). It is important to note that promoter methylation is usually a function of the history of cellular experience. Therefore, one interpretation of the reduced NF- $\kappa \mathrm{B} 1$ methylation is that NF$\kappa \mathrm{B} 1$ may have been increased at points during the learning, stimulation, and consolidation cycle. However, although day 4 $\mathrm{NF}-\kappa \mathrm{B} 1$ transcript levels differed between the VNS cortical samples $\left(\log \mathrm{FC}=-5.9, p=9.6 \times 10^{-6}\right.$, cohort 2 , no behavioral task) and the VNS hippocampal samples $(\log \mathrm{FC}=-0.4$, $p=0.08$, cohort 3 , NP task; Fig. $8 A$ ), the NF- $\kappa$ B1 DNA methylation did not reflect this difference. Instead, we found that NF- $\kappa$ B1 CpG methylation did not depend on whether rats performed the task (Fig. 8B), suggesting that the reduced methylation could instead be an independent effect of VNS.

\section{DNA DSBs in the ARC promoter are reduced by VNS}

Continuing our investigation of the link between stress-response signaling and DNA DSBs, we turned our attention to the IEG ARC. Because cortical ARC transcription was increased by VNS and correlated strongly with behavioral performance (Fig. 6B), we examined the ARC promoter for evidence of DNA DSBs, which are known to be facilitators of IEG transcription (Suberbielle et al., 2013; Madabhushi et al., 2015). Histone 2A.X phosphorylation at serine $139(\gamma \mathrm{H} 2 \mathrm{~A} . \mathrm{X})$ is known to colocalize with 
A

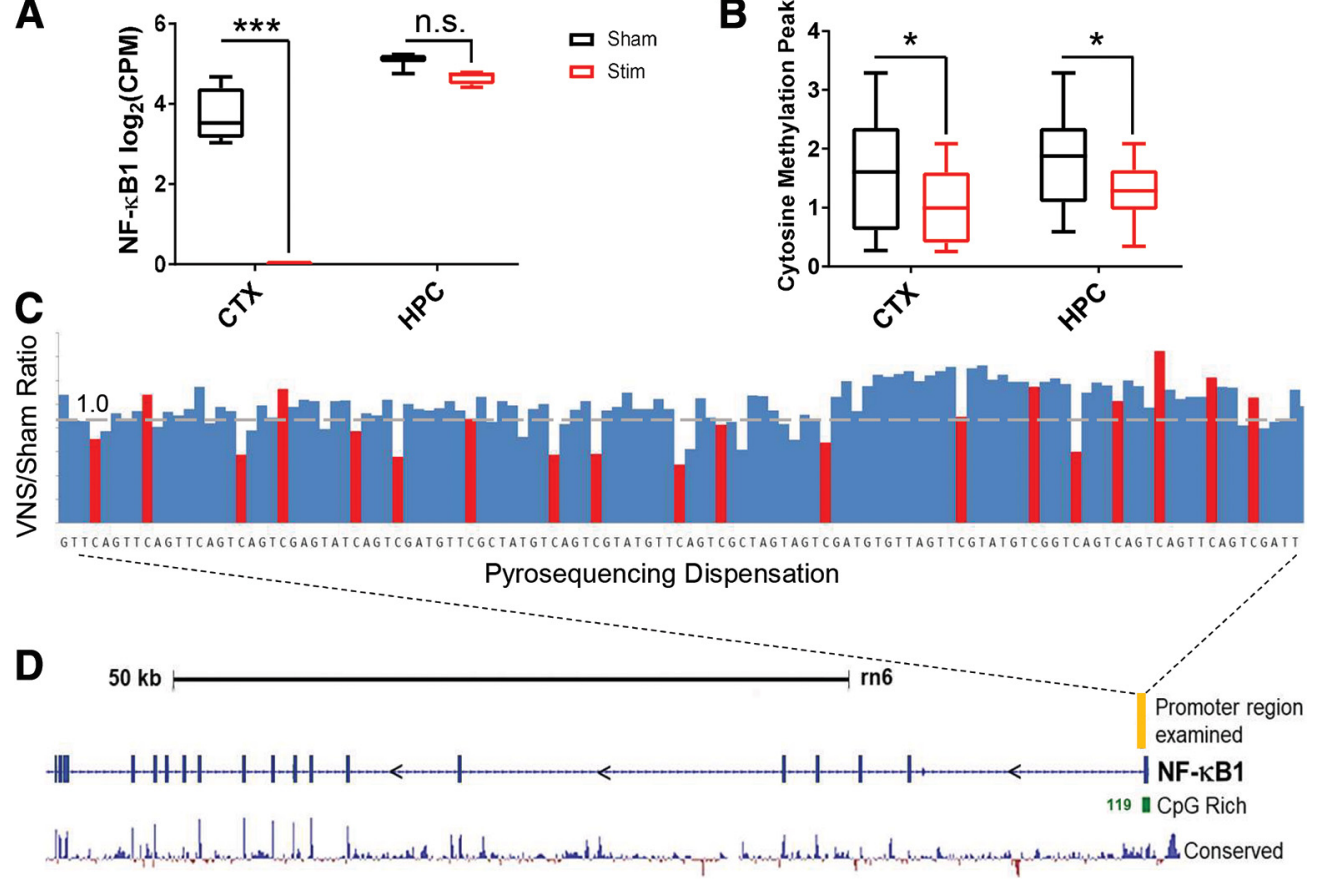

Figure 8. VNS modulates DNA methylation of NF- $\kappa B$. $A, N F-\kappa B 1$ transcripts were reduced in rats receiving VNS. $B$, Cytosine methylation within the NF- $\kappa$ B1 promoter was reduced in both cortex and hippocampus. $C$, Bar chart showing the stim versus sham ratio of occurrence for each nucleotide examined (red bars indicate methylated cytosines). Cortical NF- $\kappa B 1$ methylation in this segment was reduced by $23 \%$ overall ( $N=8 ; n=4$ sham, $n=4$ stimulated, $p=0.01$, unpaired $t$ test). $\boldsymbol{D}$, Segment of (pG-rich region examined (orange bar) in relation to exon 1 (blue) and full extent of (pG-rich region (green). ${ }^{*} p<0.05,{ }^{* * *} p<0.001$, box-and-whisker plots: central horizontal line indicates median, box indicates quartiles above and below median, whiskers indicate full range of data.

DSBs and provide a highly sensitive marker for examining DNA breaks and subsequent repair (Sharma et al., 2012). We examined $\gamma \mathrm{H} 2 \mathrm{~A} . \mathrm{X}$ both globally (through immunostaining) and locally at the promoter of ARC DNA using ChIP followed by qRT-PCR with ARC primers (cortex, cohort $2, n=8$ ). Although we did not find a significant global change in overall $\gamma \mathrm{H} 2 \mathrm{~A}$.X using immunostaining (Fig. 9C,D), ChIP revealed reduced $\gamma \mathrm{H} 2 \mathrm{~A}$.X at the ARC promoter in the cortex of VNS rats (Fig. 9B). Therefore, VNS reduced DSBs in the ARC promoter, possibly through NF- $\kappa$ B regulatory mechanisms (Krzyzaniak et al., 2011). VNSenhanced repair of DSBs was further suggested by increased transcription of genes in DNA DSB repair pathways (ASCC1, XRCC1) in the cortex $(\log \mathrm{FC}=$ $3.3, p=0.017, \log \mathrm{FC}=3.9, p=0.001)$ of VNS rats. This is consistent with our findings regarding reduced cortical NF- $\kappa \mathrm{B} 1$. However, previous research indicates that synaptic plasticity changes required for learning are accompanied by increased DSBs at IEG promoters (Madabhushi et al., 2015). Therefore, our finding of reduced promoter DSBs in the plasticity gene ARC after VNS suggests that non-monotonic changes may occur during the $4 \mathrm{~d} \mathrm{NP}$ task. This is consistent with previous findings of activity-dependent oscillations in NF- $\kappa \mathrm{B}$ and epigenetic modulators (Nelson et al., 2004; Clayton et al., 2006).
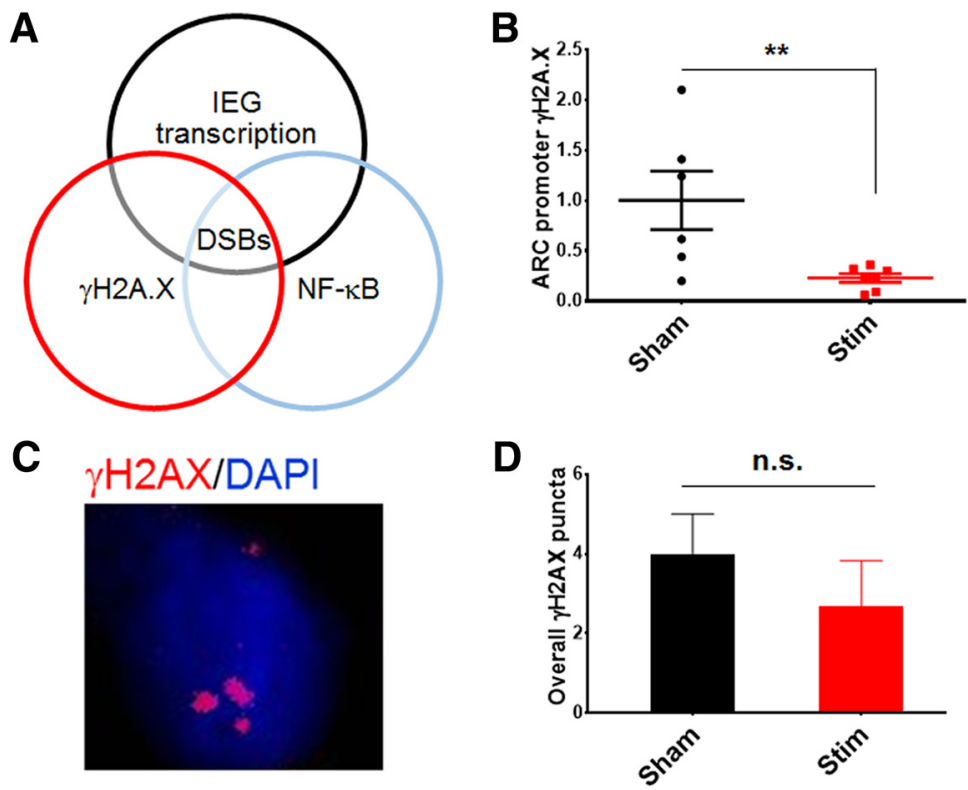

Figure 9. VNS reduces DSBs at the ARC promoter. $A, D S B s$ activate stress-response signaling (NF-kB), facilitate transcription of IEGS, and are colocated with $\gamma H 2 A . X . B$, VNS reduces $\gamma H 2 A . X$ (DSB indicator) at the promoter of IEG ARC.C, Cells stained for $\gamma H 2 A . X$ show puncta (red) in the nucleus (blue). $\boldsymbol{D}$, Quantification of global $\gamma \mathrm{H} 2 \mathrm{~A} . \mathrm{X}$ staining showed no significant difference between sham and VNS rats $(p=0.2)$. Error bars indicate SEM. $N=8\left(n=4\right.$ sham, $n=4$ stimulated). ${ }^{* *} p<0.01$.

\section{Discussion}

Novel object preference tests are popular for measuring enhanced learning and memory (Bevins and Besheer, 2006; Broadbent et al., 2010; Gaskin et al., 2010; Antunes and Biala, 2012). We designed a $4 \mathrm{~d}$ protocol to allow analysis of longer-term and short-term VNS impacts on plasticity and behavior. Objects were 
placed in alternating locations to reduce the likelihood of confounding effects due to innate preference for a particular object or location. Consistent with the expected result of VNSenhanced cognition, VNS rats preferred the novel object over the familiar object during all $3 \mathrm{~d}$ of testing, whereas sham rats, on average, spent a similar amount of time with each object.

Sham rats in our study preferred the familiar object more than most controls reported in the literature. To gain insight into this phenomenon, we reanalyzed our data to look for confounding factors. Because sham and VNS rats interacted with the novel objects for a similar amount of time, we did not find cause to implicate anxiety in the shams' lack of preference for the novel object. However, we noted that several of the objects in our study were compound/complex and thus possibly more engaging (or confusing) to the rats than the simple objects typically used. Indeed, in trials in which our simplest object (lid) was the familiar object (Fig. 1D, day 4), we found that both sham and stimulated rats preferred the novel object during the test period. Although this suggests that intrinsic object characteristics, and possibly other unknowns, may have played a role in the daily relative object preferences, the results unequivocally show that, compared with shams, VNS rats demonstrated significantly greater overall preference for novel objects.

\section{Stress-response signaling}

Reduced NF- $\kappa \mathrm{B} 1$ in the cortex and blood, along with inversely correlated hippocampal NF- $\kappa \mathrm{B} 1$ and cognitive performance, revealed a widespread VNS effect on stress-response signaling. VNS-induced reductions in NF- $\kappa \mathrm{B}$ associated with cytokine changes such as reduced TNF have been reported previously (Borovikova et al., 2000; Tracey, 2002; Andersson and Tracey, 2012). However, the minimal change in cytokine transcription in the hippocampus and cortex in our rats suggests that NF- $\kappa$ B effects in these structures could be related to alternative stress-response modulators such as hormones, histone modifiers, or DNA DSBs.

Several immune receptors correlated with reduced NF- $\kappa$ B1. Importantly, TNFRSF11B (OPG) transcripts were reduced in the cortex and hippocampus and correlated with NF- $\kappa \mathrm{B} 1$ transcription and increased novel object recognition. OPG, a cytokine receptor and negative regulator of bone resorption, is a thyroid and parathyroid hormone responsive gene. This evidence, combined with the VNS cuff proximity to the thyroid, points to potential involvement of these hormones in VNS-induced alterations to stress-response signaling, plasticity, and novel object preference.

For VNS rats that did not perform the behavioral task (cohort 2 ), reductions in NF- $\kappa \mathrm{B} 1$ transcription accumulated, leading to a complete loss of cortical NF- $\kappa \mathrm{B} 1$ transcripts. However, during the behavioral task (cohort 3), stress-response signaling likely increased as a result of exposure to novelty and then decreased by the next session of VNS, resulting in the modest cumulative NF$\kappa \mathrm{B} 1$ decrease observed in the hippocampus. Despite the large difference between transcriptional effects, VNS reduced NF- $\kappa$ B1 methylation similarly in the two groups. This suggests that DNA methylation did not drive NF- $\kappa$ B1 transcription and that reduced DNA methylation may be an independent effect of VNS. Potential mechanisms for this general demethylating effect include blood/CSF changes (suggested by reduced carbonic anhydrase transcription) and reduced TRIP10 (associated with increased methylated cytosine).

\section{Increased BDNF}

Not all of the VNS-induced transcriptional changes were linked to OPG effects. For example, BDNF transcripts and protein were increased in both the cortex and the hippocampus, but did not correlate with any of the stress-response signaling transcripts, including OPG. BDNF has been linked to successful memory consolidation (Bekinschtein et al., 2007; Lubin et al., 2008) and positive effects on treatment-resistant depression (Nemeroff et al., 2006; Aaronson et al., 2017). Consistent with previous studies reporting a link among electrical stimulation, BDNF transcription, and increased CAMK2 activity (Yan et al., 2016), we found correlations between transcription of hippocampal BDNF and learning and memory genes CAMK2A and CAMK2B. CAMK2B has been shown to facilitate ARC targeting to inactive synapses to prevent undesired enhancement of weak synapses in potentiated neurons (by reducing surface AMPA receptors) (Okuno et al., 2016).

\section{Diverse plasticity effects}

Although we observed increased BDNF and reduced OPG in both the hippocampus and cortex, overall transcription effects differed dramatically between the two structures. Investigation of a set of thyroid-responsive genes (STAT5A, FOS, HR, TXNIP; Chatonnet et al., 2015) revealed that these transcripts were reduced in the hippocampus but increased in the cortex. The cortical increase is consistent with VNS-increased thyroid hormone suggested by reduced OPG. The hippocampal decrease can be explained by the smaller reduction in OPG combined with an increase in THRA transcription because unliganded thyroid receptor represses transcription (Grøntved et al., 2015). Therefore, the dichotomy of hippocampal and cortical responses to VNS may have a common root, in part, in thyroid/OPG changes. Importantly, the different histone modifiers that correlated with reduced OPG in the cortex (KAT8 and SETD7) and hippocampus (HDAC11 and H2AFY2) likely contributed to the downstream transcriptional diversity.

ARC has been previously identified as a key synaptic plasticity gene with the capability to transfer mRNA across neurons (Bramham et al., 2008; Lonergan et al., 2010; Shepherd and Bear, 2011; Pastuzyn et al., 2018; Wall et al., 2018). In our study, ARC did not correlate with OPG, suggesting the observed thyroid/OPG effects were not primary drivers in its upregulation. Additionally, although increased BDNF has previously been shown to increase ARC (Wibrand et al., 2006), we did not find a correlation between ARC and BDNF.

The observed cortical ARC increase in VNS rats regardless of whether the cognitive task was performed suggests that VNS may induce preparatory cortical plasticity. Importantly, in rats that performed the task, cortical ARC transcription correlated with novel object preference. ARC also correlated with many epigenetic modifiers (HDAC3, DNMT3A, SETDB1, SETD7, and ANKRA2). HDAC3 is known to directly deacetylate NF- $\kappa$ B subunit Rel6 (Chen et al., 2001), causing NF- $\kappa B$ subunits to bind more strongly to I $\kappa \mathrm{B}$ in the cytoplasm. Therefore, HDAC3 upregulation may play a role in downregulated NF- $\kappa$ B1.

The increased transcriptional repression suggested by the ARC-correlating epigenetic modifiers initially appeared incongruent with the observed increased cortical transcription. However, correlation mapping suggested that repression may have been directed toward transcriptional coregulators (SIN3B), cell growth inhibitors (RTN4R, neurite outgrowth inhibitor), and metastasis enablers (TCF12, MMP17). Therefore, the positive correlation of these transcriptional repressors with ARC may be due to their role in reducing competing metabolic programs.

Additional transcriptional evidence suggests VNS may facilitate synaptic localization of $\mathrm{GABA}_{\mathrm{A}}$ receptors (increased 


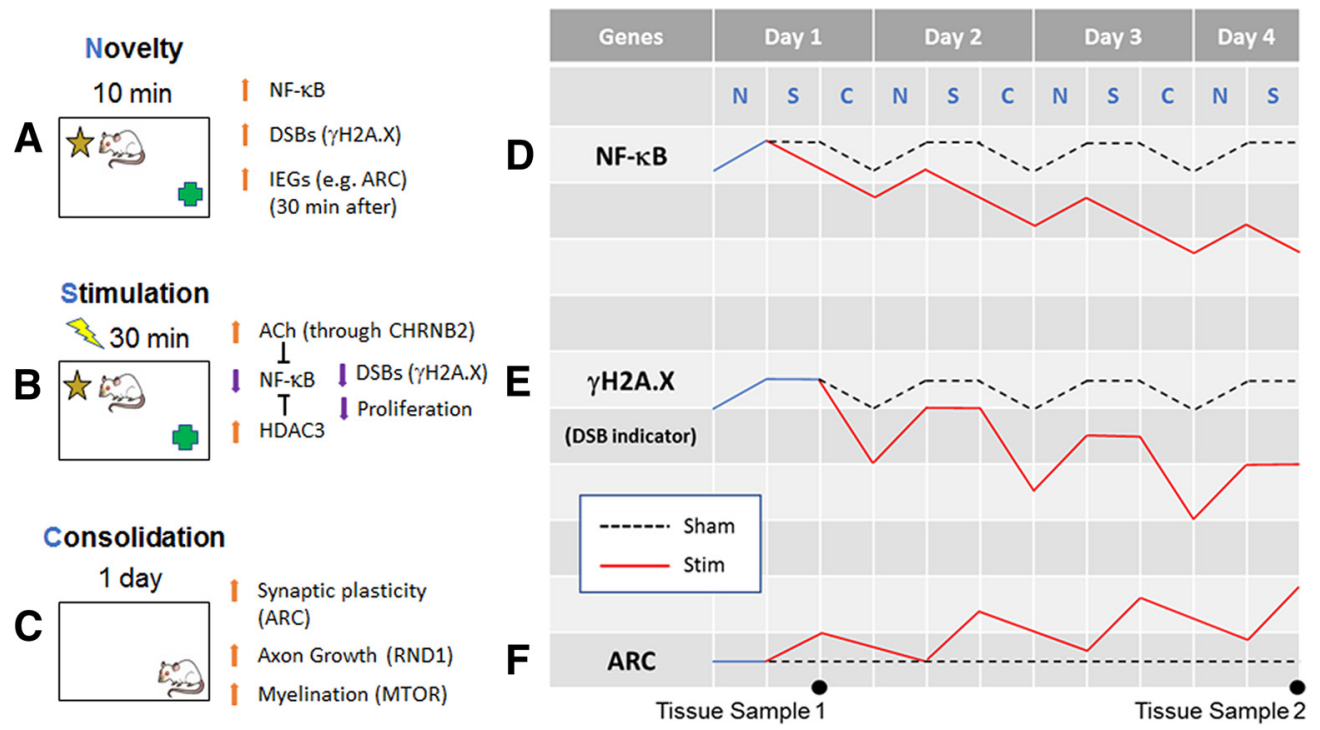

Figure 10. Working model of cortical plasticity. $A$, Novelty presentation (N) induces stress-response signaling due to DSBs in IEG promoters. $\boldsymbol{B}$, VNS (S) reduces NF- $\kappa B$ through multiple mechanisms, including increased DSB repair along with ACh and HDAC3 effects. C, Reduced NF- $\kappa$ B contributes to subsequent consolidation (C) by downregulating metabolic demand from competing activities such as proliferation and immune signaling, as well as facilitating a large pool of plasticity-enabling transcripts. Phases were repeated each day for $4 \mathrm{~d}$, leading to cumulative effects in many genes, including those shown in $\boldsymbol{D}-\boldsymbol{F}$. D, NF- $\kappa$ B is increased by exposure to novelty, but is reduced by intermittent $30 \mathrm{~Hz}$ VNS. $\boldsymbol{E}$, DSBs (indicated by $\gamma \mathrm{H} 2 \mathrm{~A} . \mathrm{X}$ ) are also increased by novelty, but rapidly repaired subsequent to VNS. $F$, Rapid DSB repair enhances cells' ability to process new cycles of transcription necessary for synaptic plasticity.

GABRG3), upregulate nicotinic cholinergic effects (CHRNB2), and increase the NMDA/AMPA ratio (increased NMDA-R subunits, GRIN2A and GRIN2D, decreased AMPA-R subunit GRIA4). Previous studies have shown these increases are implicated in enhanced cognition (Lane et al., 2010; Das et al., 2013; Kerti-Szigeti et al., 2014; Karasawa et al., 2008).

Blood IEG transcripts increased in VNS rats that did not perform the task, coinciding with cortical increases. However, rats that participated in the task displayed a decrease in IEG transcripts compared with rats that did not perform the task. This may indicate that, in the absence of learning-induced plasticity, VNS produces excess IEG transcripts (ARC and FOS in our study) that are cleared via the blood. Conversely, when IEG transcripts such as ARC are used to facilitate synaptic plasticity, fewer may be released into the blood. If this is the case, then blood ARC transcripts may be a useful measure of enhanced plasticity and learning.

Hippocampal neuronal changes that facilitate learning were also observed, such as reduced expression of potassium channels that may enable faster neuronal recovery after spiking and changes in calcium-related signaling that may alter the quantities and types of neurotransmitters released (Takács et al., 2018). Changes in blood calcium implied by downregulated OPG likely contributed to the large number of significantly changed hippocampal calcium signaling transcripts. Individual hippocampal calcium signaling transcripts correlated with histone modifiers HDAC11, H2AFY2, SETD4, and KDM4D, again suggesting involvement of epigenetic transcriptome changes in VNS-induced plasticity.

Significantly reduced hippocampal histone modifiers (HDAC11 and H2AFY2) correlated with reduced OPG and improved cognitive performance. HDAC11 is enriched in CA1, so reducing its deacetylase activity may facilitate consolidation by increasing hippocampal output to the subiculum (Broide et al., 2007), potentially through increased expression of PLEC (Fuchs et al., 2009).

\section{Stress-response signaling, plasticity, and epigenetic} mechanisms work together to enhance learning and memory Our finding of reduced $\gamma \mathrm{H} 2 \mathrm{~A}$.X in rats that received VNS without a behavioral task suggests that VNS may enhance plasticity by rapid repair of DSBs, thus facilitating quick replenishment of IEG transcripts. Enhanced DSB repair is also supported by increased cortical transcription of repair genes ASCC1 and XRCC1 and by HDAC3, which, when complexed with silencing mediator for retinoid and thyroid hormone receptor, SMRT, facilitates recovery from DNA DSBs (Yu et al., 2006). This result exemplifies the convergence of the processes we found most important for VNSenhanced cognition: neuronal plasticity (ARC), stress-response signaling (NF- $\kappa \mathrm{B} 1)$, and histone modifications $(\gamma \mathrm{H} 2 \mathrm{~A} . \mathrm{X})$.

Cycles of learning and memory initiation in the hippocampus followed by cortical consolidation (and reconsolidation) are known to be important for effective cognition (Bekinschtein et al., 2007; Lubin et al., 2008; Tay et al., 2018). A cyclical interpretation of stress-response signaling and epigenetic regulation is also supported by previous reports of oscillations in NF- $\kappa \mathrm{B}$ and related epigenetic modulators (Nelson et al., 2004; Vanden Berghe et al., 2006; Clayton et al., 2006). We constructed a proposed working model of VNS-induced cortical plasticity (Fig. 10) to illustrate how increased stress from IEG DSBs followed by VNS-induced DSB repair can cause cumulative oscillatory effects that are consistent with the observed data. This evolving landscape highlights one aspect of the difficulty inherent in optimizing VNS treatments: selecting appropriate stimulus parameters and timelines in a system that is continually adapting.

\section{Therapeutic relevance}

Our findings support previously identified VNS therapeutic effects for conditions such as arthritis (Tracey, 2002; Andersson and Tracey, 2012; Bassi et al., 2017), treatment-resistant depression (Nemeroff et al., 2006; Aaronson et al., 2017), epilepsy, and stroke (Hwang et al., 2013). Downregulated cellular proliferation and modulation of cancer-relevant genes (Hymowitz and Wertz, 
2010) suggest a potential new role for VNS in cancer treatment. The evidence also supports our study's main hypothesis that epigenetic alterations underlie VNS enhancement of cognition and plasticity. More specifically, we find that VNS induces preparatory cortical plasticity and upregulated hippocampal output that could be beneficial for treating Alzheimer's disease (Lane et al., 2010; Suberbielle et al., 2013), as well as other forms of cognitive impairment (Karvat and Kimchi, 2014; Kennedy et al., 2016). Importantly, we identified genes associated with enhanced plasticity and improved cognition that are promising independent therapeutic targets. Several targets and pathways are implicated by our discovery that OPG plays an important role in VNSinduced cognitive enhancements. Additionally, new cognitive epigenetic targets such as HDAC11, KAT8, and SIN3B are of interest because they may modulate gene expression important for enhanced learning and memory more specifically than currently prescribed broad-acting histone deacetylase inhibitors.

In conclusion, our study provides key insights into VNSinduced epigenetic alterations in pathways important for enhanced cognition. Our data reveal that VNS modulates epigenetic involvement in stress-response signaling, neuronal plasticity, and transcriptional modifications that facilitate consolidation. Our findings also identify genetic and epigenetic players in a noncanonical stress-response signaling/plasticity pathway that may be broadly important for understanding learning and memory mechanisms.

\section{References}

Aaronson ST, Sears P, Ruvuna F, Bunker M, Conway CR, Dougherty DD, Reimherr FW, Schwartz TL, Zajecka JM (2017) A 5-year observational study of patients with treatment-resistant depression treated with vagus nerve stimulation or treatment as usual: comparison of response, remission, and suicidality. Am J Psychiatry 174:640-648.

Alvarez-Dieppa AC, Griffin K, Cavalier S, McIntyre CK (2016) Vagus nerve stimulation enhances extinction of conditioned fear in rats and modulates Arc protein, CaMKII, and GluN2B-containing NMDA receptors in the basolateral amygdala. Neural Plast 2016:4273280.

Andersson U, Tracey KJ (2012) Reflex principles of immunological homeostasis. Annu Rev Immunol 30:313-335.

Antunes M, Biala G (2012) The novel object recognition memory: neurobiology, test procedure, and its modifications. Cogn Process 13:93-110.

Barrero MJ, Sese B, Kuebler B, Bilic J, Boue S, Martí M, Izpisua Belmonte JC (2013) Macrohistone variants preserve cell identity by preventing the gain of H3K4me2 during reprogramming to pluripotency. Cell Rep 3:1005-1011.

Bassi GS, Dias DPM, Franchin M, Talbot J, Reis DG, Menezes GB, Castania JA, Garcia-Cairasco N, Resstel LBM, Salgado HC, Cunha FQ, Cunha TM, Ulloa L, Kanashiro A (2017) Modulation of experimental arthritis by vagal sensory and central brain stimulation. Brain Behav Immun 64:330343.

Bekinschtein P, Cammarota M, Igaz LM, Bevilaqua LR, Izquierdo I, Medina JH (2007) Persistence of long-term memory storage requires a late protein synthesis-and BDNF-dependent phase in the hippocampus. Neuron 53:261-277.

Berridge CW, Waterhouse BD (2003) The locus coeruleus-noradrenergic system: modulation of behavioral state and state-dependent cognitive processes. Brain Res Brain Res Rev 42:33-84.

Bevins RA, Besheer J (2006) Object recognition in rats and mice: a one-trial non-matching-to-sample learning task to study 'recognition memory'. Nat Protoc 1:1306-1311.

Bolger AM, Lohse M, Usadel B (2014) Trimmomatic: a flexible trimmer for Illumina sequence data. Bioinformatics 30:2114-2120.

Borland MS, Vrana WA, Moreno NA, Fogarty EA, Buell EP, Sharma P, Engineer CT, Kilgard MP (2016) Cortical map plasticity as a function of vagus nerve stimulation intensity. Brain Stimul 9:117-123.

Borovikova LV, Ivanova S, Zhang M, Yang H, Botchkina GI, Watkins LR, Wang H, Abumrad N, Eaton JW, Tracey KJ (2000) Vagus nerve stimulation attenuates the systemic inflammatory response to endotoxin. Nature 405:458-462.

Bramham CR, Worley PF, Moore MJ, Guzowski JF (2008) The immediate early gene arc/arg3. 1: regulation, mechanisms, and function. J Neurosci 28:11760-11767.

Broadbent NJ, Gaskin S, Squire LR, Clark RE (2010) Object recognition memory and the rodent hippocampus. Learn Mem 17:5-11.

Broide RS, Redwine JM, Aftahi N, Young W, Bloom FE, Winrow CJ (2007) Distribution of histone deacetylases 1-11 in the rat brain. J Mol Neurosci 31:47-58.

Cao B, Wang J, Shahed M, Jelfs B, Chan RH, Li Y (2016) Vagus nerve stimulation alters phase synchrony of the anterior cingulate cortex and facilitates decision making in rats. Sci Rep 6:35135.

Chatonnet F, Flamant F, Morte B (2015) A temporary compendium of thyroid hormone target genes in brain. Biochim Biophys Acta 1849:122-129.

Chen Lf, Fischle W, Verdin E, Greene WC (2001) Duration of nuclear NFkappaB action regulated by reversible acetylation. Science 293:1653-1657.

Clark KB, Naritoku DK, Smith DC, Browning RA, Jensen RA (1999) Enhanced recognition memory following vagus nerve stimulation in human subjects. Nat Neurosci 2:94-98.

Clayton AL, Hazzalin CA, Mahadevan LC (2006) Enhanced histone acetylation and transcription: a dynamic perspective. Mol Cell 23:289-296.

Dai Z, Mentch SJ, Gao X, Nichenametla SN, Locasale JW (2018) Methionine metabolism influences genomic architecture and gene expression through H3K4me3 peak width. Nat Commun 9:1955.

Das I, Park JM, Shin JH, Jeon SK, Lorenzi H, Linden DJ, Worley PF, Reeves RH (2013) Hedgehog agonist therapy corrects structural and cognitive deficits in a Down syndrome mouse model. Sci Transl Med 5:201ra120 201 ra120.

Day JJ, Sweatt JD (2010) DNA methylation and memory formation. Nat Neurosci 13:1319-1323.

Day JJ, Sweatt JD (2011) Epigenetic mechanisms in cognition. Neuron 70: 813-829.

Engineer ND, Riley JR, Seale JD, Vrana WA, Shetake JA, Sudanagunta SP, Borland MS, Kilgard MP (2011) Reversing pathological neural activity using targeted plasticity. Nature 470:101-104.

Feng J, Zhou Y, Campbell SL, Le T, Li E, Sweatt JD, Silva AJ, Fan G (2010) Dnmt1 and Dnmt3a maintain DNA methylation and regulate synaptic function in adult forebrain neurons. Nat Neurosci 13:423-430.

Frankland PW, Ding HK, Takahashi E, Suzuki A, Kida S, Silva AJ (2006) Stability of recent and remote contextual fear memory. Learn Mem 13: 451-457.

Fuchs P, Zörer M, Reipert S, Rezniczek GA, Propst F, Walko G, Fischer I, Bauer J, Leschnik MW, Lüscher B, Thalhammer JG, Lassmann H, Wiche G (2009) Targeted inactivation of a developmentally regulated neural plectin isoform leads to reduced motor nerve conduction velocity. J Biol Chem 284:26502-26509.

Gaskin S, Tardif M, Cole E, Piterkin P, Kayello L, Mumby DG (2010) Object familiarization and novel-object preference in rats. Behav Processes 83: $61-71$.

Grøntved L, Waterfall JJ, Kim DW, Baek S, Sung MH, Zhao L, Park JW, Nielsen R, Walker RL, Zhu YJ, Meltzer PS, Hager GL, Cheng SY (2015) Transcriptional activation by the thyroid hormone receptor through ligand-dependent receptor recruitment and chromatin remodeling. Nat Commun 6:7048.

Haettig J, Stefanko DP, Multani ML, Figueroa DX, McQuown SC, Wood MA (2011) HDAC inhibition modulates hippocampus-dependent longterm memory for object location in a CBP-dependent manner. Learn Mem 18:71-79.

Hays SA, Khodaparast N, Hulsey DR, Ruiz A, Sloan AM, Rennaker RL 2nd, Kilgard MP (2014) Vagus nerve stimulation during rehabilitative training improves functional recovery after intracerebral hemorrhage. Stroke 45:3097-3100.

Hulsey DR, Riley JR, Loerwald KW, Rennaker RL 2nd, Kilgard MP, Hays SA (2017) Parametric characterization of neural activity in the locus coeruleus in response to vagus nerve stimulation. Exp Neurol 289:21-30.

Hwang JY, Aromolaran KA, Zukin RS (2013) Epigenetic mechanisms in stroke and epilepsy. Neuropsychopharmacology 38:167-182.

Hymowitz SG, Wertz IE (2010) A20: from ubiquitin editing to tumour suppression. Nat Rev Cancer 10:332-341.

Itzhak Y, Liddie S, Anderson KL (2013) Sodium butyrate-induced histone acetylation strengthens the expression of cocaine-associated contextual memory. Neurobiol Learn Mem 102:34-42.

Jones PA (2012) Functions of DNA methylation: islands, start sites, gene bodies and beyond. Nat Rev Genet 13:484-492. 
Karasawa J, Hashimoto K, Chaki S (2008) d-Serine and a glycine transporter inhibitor improve MK-801-induced cognitive deficits in a novel object recognition test in rats. Behav Brain Res 186:78-83.

Kaas GA, Zhong C, Eason DE, Ross DL, Vachhani RV, Ming GL, King JR, Song H, Sweatt JD (2013) TET1 controls CNS 5-methylcytosine hydroxylation, active DNA demethylation, gene transcription, and memory formation. Neuron 79:1086-1093.

Karvat G, Kimchi T (2014) Acetylcholine elevation relieves cognitive rigidity and social deficiency in a mouse model of autism. Neuropsychopharmacology 39:831-840.

Kennedy AJ, Sweatt JD (2016) Drugging the methylome: DNA methylation and memory. Crit Rev Biochem Mol Biol 51:185-194.

Kennedy AJ, Rahn EJ, Paulukaitis BS, Savell KE, Kordasiewicz HB, Wang J, Lewis JW, Posey J, Strange SK, Guzman-Karlsson MC, Phillips SE, Decker K, Motley ST, Swayze EE, Ecker DJ, Michael TP, Day JJ, Sweatt JD (2016) Tcf4 regulates synaptic plasticity, DNA methylation, and memory function. Cell Rep 16:2666-2685.

Kerti-Szigeti K, Nusser Z, Eyre MD (2014) Synaptic GABA A $_{\text {r }}$ receptor clustering without the $\gamma 2$ subunit. J Neurosci 34:10219-10233.

Krzyzaniak MJ, Peterson CY, Cheadle G, Loomis W, Wolf P, Kennedy V, Putnam JG, Bansal V, Eliceiri B, Baird A, Coimbra R (2011) Efferent vagus nerve stimulation attenuates acute lung injury following burn: the importance of the gut-lung axis. Surgery 150:379-389.

Kyono Y, Subramani A, Ramadoss P, Hollenberg AN, Bonnett RM, Denver RJ (2016) Liganded thyroid hormone receptors transactivate the DNA methyltransferase 3a gene in mouse neuronal cells. Endocrinology 157: 3647-3657.

Lane RF, Raines SM, Steele JW, Ehrlich ME, Lah JA, Small SA, Tanzi RE, Attie AD, Gandy S (2010) Diabetes-associated SorCS1 regulates Alzheimer's amyloid- $\beta$ metabolism: evidence for involvement of SorL1 and the retromer complex. J Neurosci 30:13110-13115.

Langmead B, Salzberg SL (2012) Fast gapped-read alignment with bowtie 2 . Nat Methods 9:357-359.

Levenson JM, O'Riordan KJ, Brown KD, Trinh MA, Molfese DL, Sweatt JD (2004) Regulation of histone acetylation during memory formation in the hippocampus. J Biol Chem 279:40545-40559.

Levenson JM, Roth TL, Lubin FD, Miller CA, Huang IC, Desai P, Malone LM, Sweatt JD (2006) Evidence that DNA (cytosine-5) methyltransferase regulates synaptic plasticity in the hippocampus. J Biol Chem 281:1576315773.

Lisman J, Schulman H, Cline H (2002) The molecular basis of CaMKII function in synaptic and behavioural memory. Nat Rev Neurosci 3:175190.

Livak KJ, Schmittgen TD (2001) Analysis of relative gene expression data using real-time quantitative PCR and the 2- $\Delta \Delta$ CT method. Methods 25:402-408.

Lonergan ME, Gafford GM, Jarome TJ, Helmstetter FJ (2010) Timedependent expression of Arc and zif268 after acquisition of fear conditioning. Neural Plast 2010:139891.

Lubin FD, Roth TL, Sweatt JD (2008) Epigenetic regulation of BDNF gene transcription in the consolidation of fear memory. J Neurosci 28: $10576-10586$

Madabhushi R, Gao F, Pfenning AR, Pan L, Yamakawa S, Seo J, Rueda R, Phan TX, Yamakawa H, Pao PC, Stott RT, Gjoneska E, Nott A, Cho S, Kellis M, Tsai LH (2015) Activity-induced DNA breaks govern the expression of neuronal early-response genes. Cell 161:1592-1605.

Maere S, Heymans K, Kuiper M (2005) BiNGO: a Cytoscape plugin to assess overrepresentation of gene ontology categories in biological networks. Bioinformatics 21:3448-3449.

Meadows JP, Guzman-Karlsson MC, Phillips S, Holleman C, Posey JL, Day JJ, Hablitz JJ, Sweatt JD (2015) DNA methylation regulates neuronal glutamatergic synaptic scaling. Sci Signal 8:ra61.

Miller CA, Campbell SL, Sweatt JD (2008) DNA methylation and histone acetylation work in concert to regulate memory formation and synaptic plasticity. Neurobiol Learn Mem 89:599-603.

Miller CA, Gavin CF, White JA, Parrish RR, Honasoge A, Yancey CR, Rivera IM, Rubio MD, Rumbaugh G, Sweatt JD (2010) Cortical DNA methylation maintains remote memory. Nat Neurosci 13:664-666.

Mumby DG, Gaskin S, Glenn MJ, Schramek TE, Lehmann H (2002) Hippocampal damage and exploratory preferences in rats: memory for objects, places, and contexts. Learn Mem 9:49-57.

Nelson DE, Ihekwaba AE, Elliott M, Johnson JR, Gibney CA, Foreman BE,
Nelson G, See V, Horton CA, Spiller DG, Edwards SW, McDowell HP, Unitt JF, Sullivan E, Grimley R, Benson N, Broomhead D, Kell DB, White MR (2004) Oscillations in NF-kappaB signaling control the dynamics of gene expression. Science 306:704-708.

Nemeroff CB, Mayberg HS, Krahl SE, McNamara J, Frazer A, Henry TR, George MS, Charney DS, Brannan SK (2006) VNS therapy in treatmentresistant depression: clinical evidence and putative neurobiological mechanisms. Neuropsychopharmacology 31:1345-1355.

Okuno H, Araki A, Minatohara K (2016) Inverse Synaptic Tagging by Arc. In Novel Mechanisms of Memory (Giese KP, Radwanska k, eds) pp. 99-117. Springer, Cham:Springer.

Pastuzyn ED, Day CE, Kearns RB, Kyrke-Smith M, Taibi AV, McCormick J, Yoder N, Belnap DM, Erlendsson S, Morado DR, Briggs JAG, Feschotte C, Shepherd JD (2018) The neuronal gene arc encodes a repurposed retrotransposon gag protein that mediates intercellular RNA transfer. Cell 172:275-288.e18.

Patel YA, Saxena T, Bellamkonda RV, Butera RJ (2017) Kilohertz frequency nerve block enhances anti-inflammatory effects of vagus nerve stimulation. Sci Rep 7:39810

Pinto DM, Flaus A (2010) Structure and function of histone H2AX. Subcell Biochem 50:55-78.

Robinson MD, McCarthy DJ, Smyth GK (2010) edgeR: a bioconductor package for differential expression analysis of digital gene expression data. Bioinformatics 26:139-140.

Roosevelt RW, Smith DC, Clough RW, Jensen RA, Browning RA (2006) Increased extracellular concentrations of norepinephrine in cortex and hippocampus following vagus nerve stimulation in the rat. Brain Res 1119:124-132.

Rosas-Ballina M, Olofsson PS, Ochani M, Valdés-Ferrer SI, Levine YA, Reardon C, Tusche MW, Pavlov VA, Andersson U, Chavan S, Mak TW, Tracey KJ (2011) Acetylcholine-synthesizing T cells relay neural signals in a vagus nerve circuit. Science 334:98-101.

Rosenfeld MG, Lunyak VV, Glass CK (2006) Sensors and signals: a coactivator/corepressor/epigenetic code for integrating signal-dependent programs of transcriptional response. Genes Dev 20:1405-1428.

Savell KE, Gallus NV, Simon RC, Brown JA, Revanna JS, Osborn MK, Song EY, O'Malley JJ, Stackhouse CT, Norvil A, Gowher H, Sweatt JD, Day JI (2016) Extra-coding RNAs regulate neuronal DNA methylation dynamics. Nat Commun 7:12091.

Shannon P, Markiel A, Ozier O, Baliga NS, Wang JT, Ramage D, Amin N, Schwikowski B, Ideker T (2003) Cytoscape: a software environment for integrated models of biomolecular interaction networks. Genome Res 13:2498-2504.

Sharma A, Singh K, Almasan A (2012) Histone H2AX phosphorylation: a marker for DNA damage. Methods Mol Biol 920:613-626.

Shepherd JD, Bear MF (2011) New views of arc, a master regulator of synaptic plasticity. Nat Neurosci 14:279-284

Suberbielle E, Sanchez PE, Kravitz AV, Wang X, Ho K, Eilertson K, Devidze N, Kreitzer AC, Mucke L (2013) Physiologic brain activity causes DNA double-strand breaks in neurons, with exacerbation by amyloid- $\beta$. Nat Neurosci 16:613-621.

Subramanian A, Tamayo P, Mootha VK, Mukherjee S, Ebert BL, Gillette MA, Paulovich A, Pomeroy SL, Golub TR, Lander ES, Mesirov JP (2005) Gene set enrichment analysis: a knowledge-based approach for interpreting genome-wide expression profiles. Proc Natl Acad Sci U S A 102:15545-15550.

Sun L, Peräkylä J, Holm K, Haapasalo J, Lehtimäki K, Ogawa KH, Peltola J, Hartikainen KM (2017) Vagus nerve stimulation improves working memory performance. J Clin Exp Neuropsychol 39:954-964.

Sweatt JD (2013) The emerging field of neuroepigenetics. Neuron 80:624-632

Takács VT, Cserép C, Schlingloff D, Pósfai B, Szőnyi A, Sos KE, Környei Z, Dénes Á, Gulyás AI, Freund TF, Nyiri G (2018) Co-transmission of acetylcholine and GABA regulates hippocampal states. Nat Commun 9:2848.

Takada I, Kouzmenko AP, Kato S (2009) Wnt and PPAR $\gamma$ signaling in osteoblastogenesis and adipogenesis. Nat Rev Rheumatol 5:442-447.

Tay KR, Flavell CR, Cassini L, Wimber M, Lee JL (2018) Post-retrieval relearning strengthens hippocampal memories via destabilization and reconsolidation. J Neurosci 2618-18.

Tracey KJ (2002) The inflammatory reflex. Nature 420:853-859.

Turner BM (2000) Histone acetylation and an epigenetic code. Bioessays 22:836-845.

Vanden Berghe WV, Ndlovu MN, Hoya-Arias R, Dijsselbloem N, Gerlo S, 
Haegeman G (2006) Keeping up NF- $\kappa$ B appearances: epigenetic control of immunity or inflammation-triggered epigenetics. Biochem Pharmacol 72:1114-1131.

Volcic M, Karl S, Baumann B, Salles D, Daniel P, Fulda S, Wiesmüller L (2012) NF- $\kappa$ B regulates DNA double-strand break repair in conjunction with BRCA1-CtIP complexes. Nucleic Acids Res 40:181-195.

Wall MJ, Collins DR, Chery SL, Allen ZD, Pastuzyn ED, George AJ, Nikolova VD, Moy SS, Philpot BD, Shepherd JD, Müller J, Ehlers MD, Mabb AM, Corrêa SAL (2018) The temporal dynamics of arc expression regulate cognitive flexibility. Neuron 98:1124-1132.e7

Wibrand K, Messaoudi E, Håvik B, Steenslid V, Løvlie R, Steen VM, Bramham CR (2006) Identification of genes co-upregulated with arc during BDNF-induced long-term potentiation in adult rat dentate gyrus in vivo. Eur J Neurosci 23:1501-1511.
Yan X, Liu J, Ye Z, Huang J, He F, Xiao W, Hu X, Luo Z (2016) CaMKIImediated CREB phosphorylation is involved in Ca2+ induced BDNF mRNA transcription and neurite outgrowth promoted by electrical stimulation. PLoS One 11:e0162784.

Yang X, Han H, De Carvalho DD, Lay FD, Jones PA, Liang G (2014) Gene body methylation can alter gene expression and is a therapeutic target in cancer. Cancer Cell 26:577-590.

Yu J, Palmer C, Alenghat T, Li Y, Kao G, Lazar MA (2006) The corepressor silencing mediator for retinoid and thyroid hormone receptor facilitates cellular recovery from DNA double-strand breaks. Cancer Res 66:9316-9322.

Zentner GE, Henikoff S (2013) Regulation of nucleosome dynamics by histone modifications. Nat Struct Mol Biol 20:259-266.

Zovkic IB, Guzman-Karlsson MC, Sweatt JD (2013) Epigenetic regulation of memory formation and maintenance. Learn Mem 20:61-74. 